\title{
The Binary Black Hole Model for Mrk 231 Bites the Dust
}

\author{
Karen M. Leighly ${ }^{1}$ \\ Homer L. Dodge Department of Physics and Astronomy, The University of Oklahoma, 440 W. Brooks St., \\ Norman, OK 73019 \\ Donald M. Terndrup \\ Department of Astronomy, The Ohio State University, 140 W. 18th Ave., Columbus, OH 43210 \\ Sarah C. Gallagher \\ Department of Physics \& Astronomy and Centre for Planetary and Space Exploration, The University of \\ Western Ontario, London, ON, N6A 3K7, Canada \\ Adrian B. Lucy \\ Department of Astronomy, Columbia University, 550 W. 120th Street, New York, NY 10027
}

\begin{abstract}
Mrk 231 is a nearby quasar with an unusually red near-UV-to-optical continuum, generally explained as heavy reddening by dust (e.g., Leighly et al. 2014). Yan et al. (2015) proposed that Mrk 231 is a milli-parsec black-hole binary with little intrinsic reddening. We show that if the observed FUV continuum is intrinsic, as assumed by Yan et al. (2015), it fails by a factor of about 100 in powering the observed strength of the near-infrared emission lines, and the thermal near and mid-infrared continuum. In contrast, the line and continuum strengths are typical for a reddened AGN spectral energy distribution. We find that the He $\mathrm{I}^{*} / \mathrm{P} \beta$ ratio is sensitive to the spectral energy distribution for a one-zone model. If this sensitivity is maintained in general broad-line region models, then this ratio may prove a useful diagnostic for heavily reddened quasars. Analysis of archival HST STIS and FOC data revealed evidence that the farUV continuum emission is resolved on size scales of $\sim 40$ parsecs. The lack of broad absorption lines in the far-UV continuum might be explained if it were not coincident with the central engine. One possibility is that it is the central engine continuum reflected from the receding wind on the far side of the quasar.
\end{abstract}

Subject headings: accretion — quasars: emission lines — quasars: individual (Mrk 231) — quasars: supermassive black holes

\section{Introduction}

The confirmed existence of a milli-parsecseparation supermassive black hole $(\mathrm{SMBH})$ bi-

\footnotetext{
${ }^{1}$ Visiting Astronomer at the Infrared Telescope Facility, which is operated by the University of Hawaii under Cooperative Agreement no. NNX-08AE38A with the National Aeronautics and Space Administration, Science Mission Directorate, Planetary Astronomy Program.
}

nary would be an important discovery. SMBH binaries may be present in nature as a consequence of hierarchical mergers of dark matter halos, so the incidence of binary AGN provides a potentially important test of galaxy assembly models. They may be a strong source of gravitational wave emission, and are therefore potentially important probes of general relativity. 
Mrk 231 is a well-known, nearby $(z=0.0421)$ ultra-luminous infrared galaxy that has a Seyfert 1 optical spectrum (Sanders et al. 1988). The infrared emission is thought to be a combination of AGN and starburst activity (e.g., Farrah et al. 2003, and references therein). Recently, attention has been again drawn to this galaxy after the discovery of a powerful, wide-angle, kiloparsec-scale molecular outflow (Rupke \& Veilleux 2011).

Mrk 231 has an unusual spectral energy distribution (SED). While the optical through infrared SED appears typical of quasars, the spectrum is strongly cut off through the near UV, and the continuum is very weak toward shorter wavelengths. The near-UV-through-infrared SED was studied by Leighly et al. (2014). We found that the unusual shape was consistent with circumstellar reddening, which is distinguished by a large covering fraction, and large optical depths, approaching one, that produce increased extinction in the blue and UV, with red light scattered back into the line of sight as a secondary effect. This type of reddening has been observed in Type 1a supernovae (Wang 2005; Goobar 2008), and in Gammaray Burst afterglows (Fynbo et al. 2014). Some of the theory was developed to treat the general case of the transfer of radiation in galaxies (Witt et al. 1992). In a systematic study of quasar reddening performed by Krawczyk et al. (2015), while most SEDs were best fit by a SMC reddening curve, a few were better fit by the circumstellar one. This type of reddening is natural when the geometry is predominately spherical, rather than a screen as usually assumed. Veilleux et al. (2013) also interpreted the unusual near-UV-through-optical spectral energy distribution in terms of reddening, although their proposed reddening mechanism was somewhat different.

Yan et al. (2015) proposed an alternative explanation of the unusual SED. They suggest that Mrk 231 hosts a milli-parsec binary black hole system, with nearly negligible reddening. The smallermass black hole $\left(4.5 \times 10^{6} \mathrm{M}_{\odot}\right)$ accretes as a thin disk and dominates the weak UV emission. The larger-mass black hole $\left(1.5 \times 10^{8} \mathrm{M}_{\odot}\right)$ has a low accretion rate and radiates inefficiently as an Advection Dominated Accretion Flow (ADAF). These two black holes are surrounded by a circumbinary disk, which dominates the optical and IR, and the steep rolloff observed toward the UV is the inner edge of the circumbinary disk. Moreover, they suggest that a steep rolloff from the optical toward the UV is a characteristic signature of binary black holes, and thus finding objects with similar spectra provides a method to discover these objects. A not-to-scale schematic diagram of the model is shown in Fig 1 see also Yan et al. (2015) Fig. 1.

In this paper, we critique the binary black hole model for Mrk 231 presented by Yan et al. (2015); in particular, we examine whether the binary black hole model can produce the observed emission lines. In 92 , we review the features and assumptions of the Yan et al. (2015) model relevant to the production of the emission lines. 3 motivates our use of near-infrared emission lines, combined with C IV, as diagnostics, and describes the data that we compiled. 44 lays out the several spectral energy distributions used for simulations. $\$$ [5 reports the results obtained using a set of one-zone Cloudy models. 6 describes the analysis of archival HST STIS and FOC data indicating evidence for extended emission in the far UV. \$7 summarizes our results: we find that the binary black hole model cannot produce the observed emission lines. We also show that the binary black hole model cannot power the observed mid-infrared continuum (torus) emission. We briefly discuss the potential utility of the infrared emission lines for constraining the intrinsic spectral energy distribution in obscured quasars, as well as a possible reflection origin for the far UV continuum.

\section{The Yan et al. (2015) Model}

A critical feature of the Yan et al. (2015) model is that only the emission from accretion onto the small mass black hole contributes significantly to the photoionizing continuum. They identify the continuum of the small-mass black hole with the weak far-UV continuum emission Veilleux et al. 2013), while the near-UV through infrared emission originates in the circumbinary disk. Thus, their model requires that the photoionizing flux of a small-mass black hole power the line emission of a quasar with a combined mass of $\sim 2$ orders of magnitude larger. As we will show in 3.2 if this were true, then the observed nearinfrared lines would be required to exhibit equivalent widths with respect to the photoionizing continuum (rather than the observed continuum) 

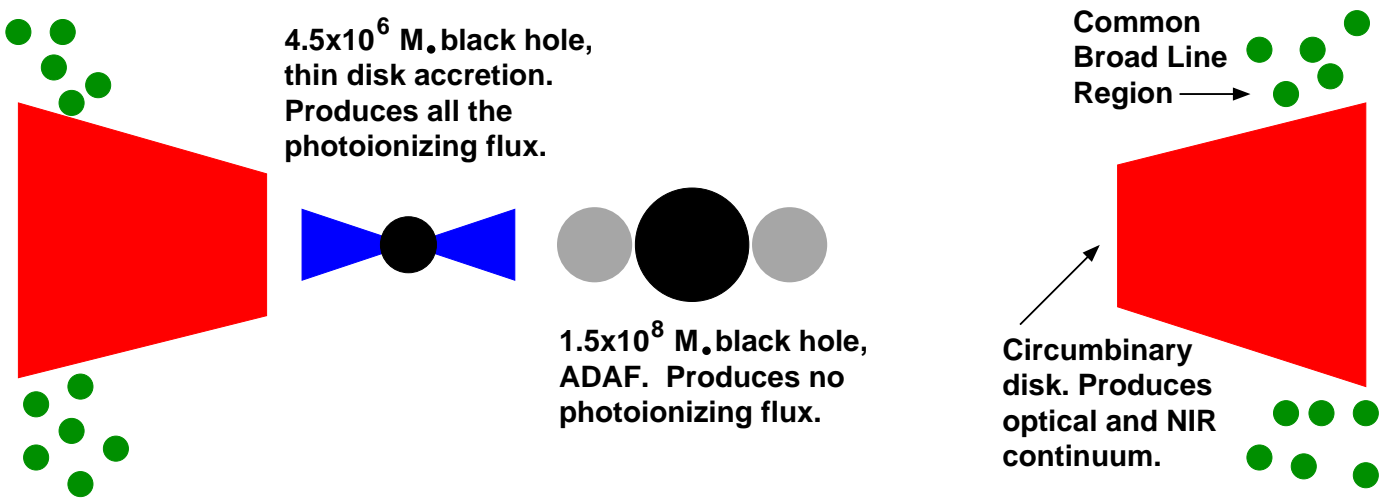

Fig. 1.- A schematic diagram of the Yan et al. (2015) binary black hole model for Mrk 231; see also Yan et al. (2015, Figure 1) for a face-on view. The smaller $4.5 \times 10^{6} \mathrm{M}_{\odot}$ black hole accretes as a thin disk and radiates efficiently, thereby emitting all the photoionizing flux that powers the broad-line region emission, while the larger $1.5 \times 10^{8} \mathrm{M}_{\odot}$ black hole accretes at a low rate, as an ADAF, and thus radiates inefficiently, producing no significant photoionizing flux. The BLR is predicted to be about 1.5 times the radius of the inner edge of the circumbinary disk, and is required emit at more than 100 times the flux of a typical BLR to produce the observed near-infrared emission lines.

about 100 times larger than normal.

Yan et al. (2015) make assumptions about the spectral energy distribution of the small mass black hole that result in a relatively high fraction of its emission emerging in the far UV. Inferred to be accreting at a significant fraction of Eddington, the small black hole's accretion disk would be expected to be bright, and have a high inner-edge temperature. They assume that the optical-UV continuum is very blue, having the slope of the sum-of-blackbodies accretion disk, i.e., $F_{\nu} \propto \nu^{1 / 3}$. In contrast, quasars are generally observed to have a redder optical-UV continuum, closer to $F_{\nu} \propto \nu^{-0.5}$; this is one of the unexplained mysteries of accretion disks, along with the lack of polarization and clear evidence for Lyman edges (Koratkar \& Blaes 1999). Finally, they assume that the X-ray flux is negligible. This is inconsistent with observations, as we show in 4 . These factors combine to make their assumed accretion disk emit a maximally high fraction of its continuum in photoionizing photons. We explore the effect of their optimized spectral energy distribution the predicted line emission in 95 , along with the predicted emission using more typical SEDs, and show that none of them can produce the observed flux and ratios of the near-infrared helium and hydrogen broad lines.
Yan et al. (2015) address the question of line emission in their $\S 6$. They make a simple nebular approximation and compute the expected number of $\mathrm{H} \alpha$ photons resulting from recombination of hydrogen in a gas illuminated by their assumed spectral energy distribution. They predict that sufficient $\mathrm{H} \alpha$ emission would be produced if the global covering fraction is $\Omega \sim 0.5$. However, this analysis is insufficient, because hydrogen lines are produced in the partially-ionized zone in quasars, and the line ratios and fluxes are observed to be different from those predicted using the simple nebular approximation (e.g., Osterbrock \& Ferland 2006; Kwan \& Krolik 1981; Davidson \& Netzer 1979). Also, quasars produce other emission lines besides the Balmer lines, and it is not obvious that those would be produced with sufficient strength to match observations. We perform a more realistic and complete line analysis in $\$ 5$.

\section{Data}

\subsection{Emission Lines Considered}

Our goal in this paper was to determine whether the line emission observed in Mrk 231 is consistent with the binary hypothesis put forth by Yan et al. (2015), not to provide a full model of the emission lines. Therefore we considered just a 
few lines that provide sufficient diagnostic power.

The near infrared spectrum is relatively free of the effects of reddening, regardless of the interpretation of the continuum. Our spectrum was obtained using the SpeX instrument on the IRTF, and the details of the observation are found in Leighly et al. (2014). We use He I* $\lambda 10830$, a line that arises from recombination of once-ionized helium. The energy required to create $\mathrm{He}^{+}$is $24 \mathrm{eV}$, and therefore He I* probes the H II part of the broad-line region emission. Being a recombination line, it is principally a diagnostic of the flux of the ionizing continuum on the broad-line-region gas. For example, for a semi-infinite slab, the flux of this line is monotonic with the helium-continuum photoionizing flux.

We also used Paschen $\beta$ at $12818 \AA$ and Paschen $\alpha$ at $18751 \AA$. Paschen $\beta$ occurs in close proximity to the He I line in the spectrum, so reddening does not affect their line ratios significantly. The ratio of Paschen $\alpha$ to Paschen $\beta$ can be influenced by reddening, but much less than, for example, the Balmer lines, since reddening curves flatten toward the near infrared.

The Paschen lines are recombination lines of hydrogen, and their fluxes and ratios are influenced strongly by the physical conditions of the line-emitting gas. Although these lines are produced throughout the ionized gas slab, a significant amount is produced beyond the hydrogen ionization front, in the partially-ionized zone. Our simulations (5) show that a significant column of partially-ionized gas is required for these lines to be observable against the bright quasar continuum. In the partially-ionized zone, the opacity to Lyman lines can be very large, and hydrogen line ratios are dramatically different from those predicted in the simple nebular approximation (e.g., Osterbrock \& Ferland 2006; Kwan \& Krolik 1981). For example, absorbed and thermalized Ly $\alpha$ can create a significant population of hydrogen in $n=2$, which can then suffer photoionization by photons with wavelengths shorter than $3646 \AA$. X-ray photoionization is also important. In addition, while hydrogen in $n=1$ cannot be collisionally excited because the difference in energy between $n=1$ and $n=2$ is too large, hydrogen in $n=2$ can experience collisional excitation, and this process will contribute to the Balmer and Paschen lines. The presence of a population of hydrogen in $n=2$ means that Balmer lines will also experience significant optical depths, reducing the radiative de-excitation and cooling. Additional processes such as charge exchange and collisional de-excitation may also contribute. Turbulence or differential velocities will change the line optical depth, further altering the line ratios (e.g., Bottorff et al. 2000). This means that the recombination line fluxes and ratios are best estimated using a photoionization code such as Cloudy, which accounts for all of these processes.

We use C IV $\lambda \lambda 1548,1551$ to probe the UV where reddening is important. C IV is a collisionallyexcited line that is produced in the H II portion of the broad-line region. It is generally one of the strongest lines present in quasar spectra, a consequence of the relatively high abundance of carbon, and its easy excitability, as $\mathrm{C}^{+3}$ has only one valence electron.

In this paper, we use as our diagnostics the strength of the He I* line (either the equivalent width of the line, or the predicted flux, depending on the situation; see below), and the line flux ratios $\mathrm{He} \mathrm{I}^{*} / \mathrm{P} \beta, \mathrm{P} \alpha / \mathrm{P} \beta$, and $\mathrm{He} \mathrm{I}^{*} / \mathrm{C}$ IV. Thus, we are leaving out a great deal of physics known to be relevant to the broad line region. For example, we do not take into account the fact that the BLR is not likely to be characterized by a single set of physical parameters (ionization parameter, density, column density) but rather to be a superposition of line emission from gas characterized by a range of parameters. This latter case is addressed by the Locally Optimally Emitting Clouds (LOC) models (Baldwin et al. 1995), and LOC models for some of the lines considered in this paper have been investigated by Ruff et al. (2012). In this paper we consider a one-zone photoionization model, principally because it is sufficient to prove our point, as the effect that we see is very apparent, even without photoionization analysis, as described in 3.2

It is also known that various emission lines can have different shapes that reflect an origin in kinematically different gases. For example, in some relatively rare cases, the high-ionization lines such as C IV can be broad and blueshifted, while intermediate- and low-ionization lines are narrow and symmetric about the rest wavelength (e.g., Leighly 2004). We do not address this in our analysis either, again because the result we find is not 
subtle, and because the observed line profiles don't warrant this consideration.

Finally, emission lines are known to respond to the shape of the spectral energy distribution (SED). If the SED is hard, i.e., the $\mathrm{X}$-ray band is strong relative to the UV, then highionization lines are observed to be strong (e.g., Casebeer et al. 2006). If the SED is soft, then the high-ionization lines are observed to be weak (e.g., Leighly et al. 2007a). We address this effect by considering several spectral energy distributions that to first approximation span the range of shapes observed in AGN and quasars.

\subsection{Mrk 231}

The He $\mathrm{I}^{*}, \mathrm{P} \beta$, and $\mathrm{P} \alpha$ lines were modeled in Leighly et al. (2014), and we take those measurements from that analysis.

HST has observed the C IV region twice, and both observations are available in the archive. The first observation was taken in 1996 with FOS and is shown in Gallagher et al. (2002). The second one was taken in 2014 using COS. They are consistent with one another, although the COS spectrum has much better signal-to-noise ratio. We measure the flux in the C IV line in the COS spectrum by fitting it with two Gaussians.

Analysis of the near-infrared line equivalent widths shows us, in a simple qualitative way, why the Yan et al. (2015) model is untenable. It is known that the properties of the broad line emission among AGN and quasars (e.g., the lines observed, their equivalent widths, and their ratios) are roughly the same over a factor of $\sim 10,000$ in inferred black hole masses and luminosities. Some variation does occur, for example, the Baldwin effect (Baldwin et al. 1995), but this accounts for a variation of less than one order of magnitude in equivalent width over four orders of magnitude in luminosity (e.g., Dietrich et al. 2002, Fig. 7). The constancy of the line equivalent widths means that photoionizing flux scales with the continuum under the emission lines. Thus, luminous quasars have luminous BLR emission, and less luminous AGN have less luminous BLR emission. So it is not reasonable to expect that a small black hole will be able to power the line emission of a luminous quasar.

This idea is investigated qualitatively in Fig. 2] which shows a variation of Leighly et al. (2014) Figure 5 that includes analysis of the emission lines. We first overlaid the intrinsic continuum, inferred in Leighly et al. (2014), with an opticalIR composite spectrum created by joining the SDSS composite (Vanden Berk et al. 2001) to the SDSS/IRTF composite Glikman et al. 2006) at around $4000 \AA$. The merged composite provides infrared coverage, and avoids the well-known longwavelength flattening present in the SDSS quasar composite due to host galaxy contributions of lower-luminosity quasars that dominate the SDSS composite on the red end of the visible band. The match is good except at very long wavelengths where an additional hot blackbody component is present (Leighly et al. 2014).

Next, we applied the Goobar (2008) reddening curve, using the parameters that we inferred in Leighly et al. (2014), to the quasar composite spectrum. Overlaid is the observed Mrk 231 spectrum. The overall agreement is very good through the optical and near UV where the line emission is typical of quasars. Mrk 231 has somewhat stronger Fe II emission, and somewhat weaker Balmer emission, but the difference is within the range observed among AGN and quasars. The reddened composite spectrum also provides a reasonable match to the near-UV continuum, against which the Mg II and Fe II broad absorption lines expected in this FeLoBAL can be seen. We discuss the far-UV in 8 .

The Yan et al. (2015) model posits that the continuum from the normally-accreting small black hole is swamped by the circumbinary disk emission through the optical and infrared, but it becomes visible in the UV, shortward of the rolloff caused by the inner edge of the circumbinary disk. The gray dashed line in Fig. 2 shows the Richards et al. (2006) continuum scaled to match the near-UV spectrum; this represents the putative continuum of the small black hole. In the region of the infrared lines of interest, around 1 micron, this continuum is a factor of $\sim 100$ below the observed Mrk 231 continuum. Thus, if the small black hole is producing all of the photoionizing photons in the system, then the infrared line equivalent widths would have to be a factor of $\sim 100$ stronger than typical with respect to the small black hole continuum in order to show the observed equivalent widths with respect to the ob- 


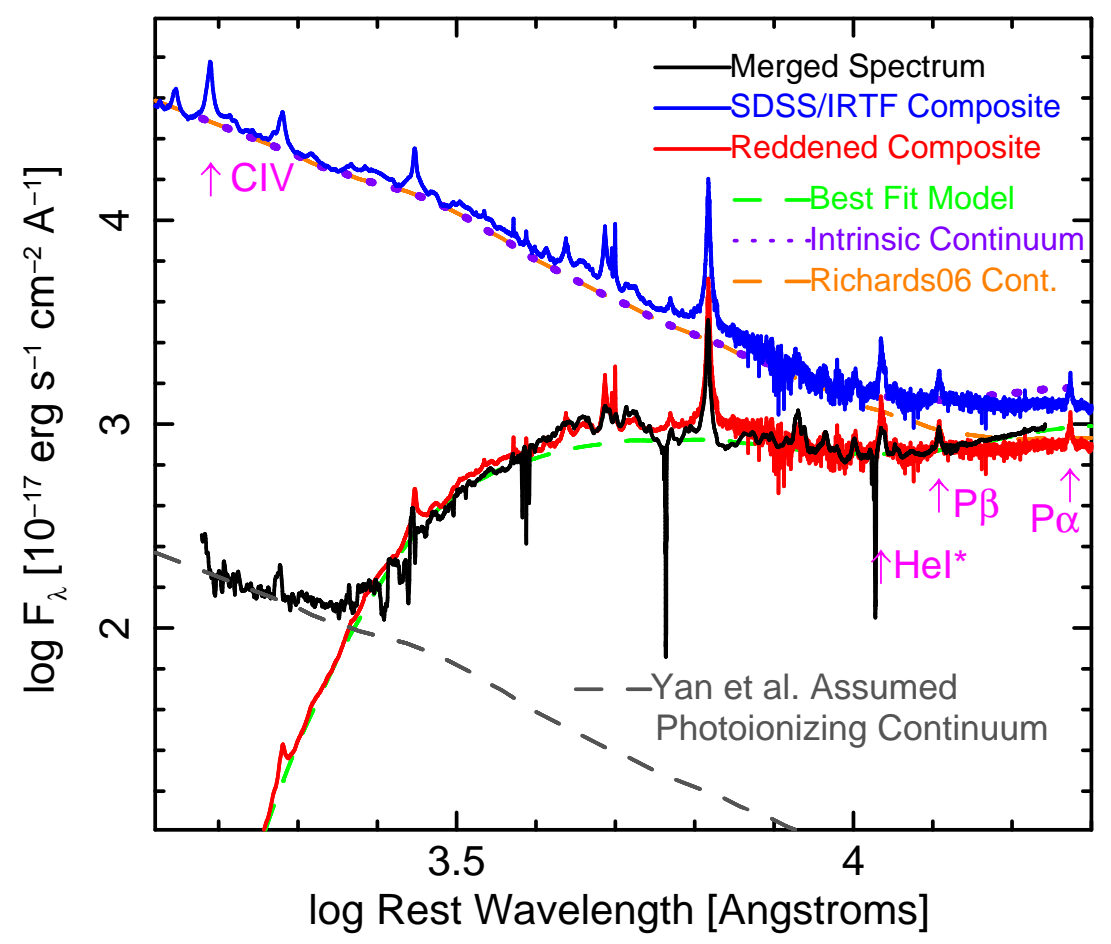

Fig. 2.- Adapted from Leighly et al. (2014) Figure 5. We show the observed broad-band spectrum from Mrk 231 (black) overlaying the best-fitting continuum model (dashed green) developed using the Richards et al. (2006) continuum (dashed orange) plus a blackbody component from hot dust, and subject to the circumstellar reddening; see Leighly et al. (2014) for details. Overlaid is a composite spectrum created merging the SDSS quasar composite (Vanden Berk et al. 2001) and the IRTF quasar composite (Glikman et al. 2006) in solid blue, and subject to the same reddening, in solid red; see Section 3.2 for details. This yields a good fit over most of the bandpass, except for the continuum shortward of $\sim 2200 \AA$; this is a separate component that may arise from scattering (\$7.6). We also overlay the Richards et al. (2006) continuum scaled to match the continuum flux at $2000 \AA$ (dashed gray). In the Yan et al. (2015) model, this taken to be the emission from the accretion disk of the small-mass black hole. The wavelengths of the four diagnostic lines used in this paper are marked.

served continuum. This does not seem plausible, as such huge equivalent widths have never been seen. Indeed, as we will show in $\$ 5.2$, the smaller black hole continuum lacks sufficient power to produce the observed infrared line emission.

We used the inferred luminosities to estimate the radius of the broad line region. Interpolating the flux density at $5100 \AA$ from the Leighly et al. (2014) intrinsic continuum and the Yan et al. (2015) photoionizing continuum (orange and gray lines in Fig. 20, and using the "clean" parameters for the radius/luminosity relationship from Bentz et al. (2013), the $\mathrm{H} \beta$ emitting BLR is es- timated to be located 99 and 6.5 light days from the central engine for the reddened and the binary black hole interpretations, respectively. The first value seems typical for a quasar, while the second value is small among reverberation-mapped AGN (Bentz et al. 2013). Relatively rapid variability of $\mathrm{H} \beta$ would be predicted; that has never been reported (see also Veilleux et al. 2016). Moreover, the inner edge of the circumbinary disk is estimated by Yan et al. (2015) to be 4.2 light days from the center. So, in the Yan et al. (2015) scenario, the BLR would have to be located on top of the circumbinary disk, close to its inner edge, and, 
as discussed above, would have to emit more than 100 times the normal flux of a typical broad-line region.

\subsection{Comparison Sample}

In order to interpret the line emission from Mrk 231, we compiled the characteristic properties of a small sample of Seyfert galaxies and quasars for comparison. Infrared spectra of nearby Seyfert galaxies and quasars were presented by Landt et al. (2008), and we took He I*, $\mathrm{P} \beta$, and $\mathrm{P} \alpha$ (when available) line fluxes from this reference. We excluded Mrk 590 because it has turned into a Seyfert 2 (Denney et al. 2014). We excluded NGC 3227 as it is highly reddened (Crenshaw et al. 2001). Our sample included 15 objects with measurements of these three lines and C IV from the literature (see below), and three more objects with measurements of He I*, $\mathrm{P} \beta$, and $\mathrm{C}$ IV only. Among these 18 objects, 14 have black hole masse:2. The range of log black hole masses represented, in units of solar mass, is 6.88 to 8.84 , with a mean of 7.66 and standard deviation of 0.54 . The range therefore is a bit higher than the log black hole mass inferred for the small mass black hole (6.65 [solar masses]). However, we found that two objects with small log black hole masses, NGC 4051 (6.130) and NGC 4748 (6.41), have infrared line properties (Riffel et al. 2006) consistent with the range of our comparison sample.

Landt et al. (2008) attempted to deconvolve the emission lines in terms of a broad line and a narrow line. This is an uncertain procedure unless there is a break in slope between the broad and narrow line (i.e., as in a Seyfert 1.5 or 1.8); in general, it is difficult to determine how much of the line should be ascribed to the narrow line region, especially when the line is cuspy. To avoid this uncertainty, we used the total line flux, i.e., the sum of the narrow and broad line fluxes in Landt et al. (2008) Table 5. We note that the narrow line flux is generally much smaller than the broad line flux (the median ratio of narrow to broad line flux is about 9\%), so this approximation does not increase the uncertainty significantly. Moreover, we used the line ratios of the comparison sample simply as an indication of the range of

\footnotetext{
${ }^{2}$ http://www.astro.gsu.edu/AGNmass/
}

ratios observed in nature, so high precision is not required.

Reddening can alter the line ratios, but to different degrees depending on the ratio. Reddening influences the $\mathrm{He} \mathrm{I}^{*} / \mathrm{P} \beta$ ratio minimally for typical objects; for example, a modest $E(B-V)=0.1$ and an SMC reddening curve results in a decrease in this ratio by about $2.3 \%$. The difference is larger for a heavily-reddened object like Mrk 231, where the Goobar (2008) reddening curve with the parameters measured by Leighly et al. (2014) yield a decrease in the ratio of $8.3 \%$. Reddening is somewhat more important for the $\mathrm{P} \alpha / \mathrm{P} \beta$, where $E(B-V)=0.1$ increases the ratio by $3.4 \%$ for SMC, and $19 \%$ for the reddening measured in Leighly et al. (2014). Reddening is much more important for the He I*/C IV ratio, which increases by $290 \%$ for $E(B-V)=0.1$, i.e., a factor of 126 times larger effect than for the $\mathrm{He} \mathrm{I}^{*} / \mathrm{P} \beta$ ratio. So, we think that much of the origin of the large range of $\mathrm{He} \mathrm{I}^{*} / \mathrm{P} \beta$ ratios is intrinsic, rather than a consequence of reddening, because the dispersion, parameterized by the standard deviation divided by the mean, is similar for the $\mathrm{He} \mathrm{I}^{*} / \mathrm{P} \beta$ ratio $(0.37)$ and the He I/C IV ratio (0.81). If reddening were dominating the range of observed $\mathrm{He} \mathrm{I}^{*} / \mathrm{P} \beta$ ratios, then a much larger dispersion would be expected for the He I/C IV ratio.

We also included data from the intrinsically Xray weak quasar PHL 1811 (Leighly et al. 2001, 2007a,b). Veilleux et al. (2016) draw comparisons with this object, positing that Mrk 231 has some similarities with PHL 1811 analogs and weak-lined quasars. We analyzed a spectrum from our observations made using IRTF SpeX on 2008 August 22,23 , and 24 . We fit the continuum with a polynomial, and the emission lines with Lorentzian profiles in the He $\mathrm{I}^{*} / \mathrm{P} \beta$ region, requiring that the Paschen lines (i.e., the isolated $\mathrm{P} \beta$ line and the blended $\mathrm{P} \gamma$ line) have the same width and a separation based on rest wavelengths. Several Fe II lines were modeled with Gaussians. We model the self-absorption on the He I* emission line using a Gaussian optical depth profile. $\mathrm{P} \alpha$ is relatively isolated and was well modeled with a Lorentzian profile.

We obtained C IV measurements from the literature. We dropped IRAS 1750+508, H1934-063, and H 2106-099 from the sample because they had had no UV spectroscopic observations. We 
extracted as many measurements as possible from Kuraszkiewicz et al. (2002), Kuraszkiewicz et al. (2004), and/or Tilton \& Shull (2013) because they presented a uniform analysis of large sets of HST spectra. Other sources of HST measurements included O'Brien et al. (2005) for PDS 456, Laor et al. (1994) for 3C 273 and H1821+643, Kriss et al. (2000) for NGC 7469, and Leighly et al. (2007b) for PHL 1811. There were only IUE measurements available for Mrk 79, PG 0844+349, Mrk 110 and NGC 4593, compiled in Wang et al. (1998). These were used with caution because comparison of values from the subset of objects that also had observations using HST showed that the Wang et al. (1998) measurements were consistently a factor of $\sim 10$ lower. We suspected that the flux-units footnote on Wang et al. (1998) Table 1 is too low by a factor of 10 , and we therefore multiplied their values by 10 .

The UV spectroscopic observations were not made simultaneously with the infrared spectroscopic observations, so relative variability is a concern. We estimated the importance of this effect by compiling C IV measurements from multiple $H S T$ observations when available: these are shown by blue and green points in Fig. 3. Examination of this figure shows that the $\mathrm{He} \mathrm{I}^{*} / \mathrm{C}$ IV ratio varies more across the sample than it does for any single object (quantified below), and therefore relative variability is not important. Again, our intention was to estimate the range of the $\mathrm{He} \mathrm{I}^{*} / \mathrm{C}$ IV ratio observed in nature, and high precision was not necessary.

\subsection{Observed Ranges of Line Properties}

We derived the range of line properties observed in the comparison sample. These ranges were used to compare with those from Mrk 231, and to constrain the simulations discussed in $\$ 5$. Fig. 3 shows the ratios $\mathrm{He} \mathrm{I}^{*} / \mathrm{P} \beta, \mathrm{P} \alpha / \mathrm{P} \beta$, and $\mathrm{He} \mathrm{I}^{*} / \mathrm{C}$ IV as a function of luminosity in the He $\mathrm{I}^{*}$ line.

The $\mathrm{He} \mathrm{I}^{*} / \mathrm{P} \beta$ ratio is shown in the top left panel. This ratio lies between 0.37 and 2.52 (a range of a factor of $\sim 7$ ), with mean and standard deviation of $1.67 \pm 0.61$. For Mrk 231, we show the observed ratio, and the ratio after correcting for the reddening inferred in Leighly et al. (2014). Neither Mrk 231 nor PHL 1811 have exceptional values of this ratio. To constrain the simulations, we use an upper limit of 2.5 (as ob- served) but extend the lower limit to 0.1 in order to roughly compensate for possible contribution of He I* emission from an outflowing component that may not be present in $\mathrm{P} \beta$.

The $\mathrm{P} \alpha / \mathrm{P} \beta$ ratio is shown in the middle panel. This ratio lies between 0.94 and 1.74 (a factor of 1.84), with mean and standard deviation of $1.20 \pm 0.21$ for the comparison objects. The observed ratio for Mrk 231 is slightly high compared with this range (1.79); however, when corrected for the reddening curve inferred by Leighly et al. (2014), the ratio drops to 1.58, a value roughly consistent with that of the comparison sample. To constrain the simulations, we consider values between 0.9 and 2.0 .

As discussed in Osterbrock \& Ferland (2006), in the low-density, low-optical-depth limit (Case A), and the large-optical-depth limit whereby every Lyman-line photon is scattered many times (Case $\mathrm{B}), \mathrm{P} \alpha / \mathrm{P} \beta$ ratios of 2.33 and 2.28 are predicted, respectively. Our ratios are significantly lower than that, suggesting high optical depths in the broad line region.

The range of observed He I*/C IV ratios is large in the comparison sample (0.025 to 0.37 , a factor of $\sim 15$ ), with a mean of 0.11 and a standard deviation of 0.089 . PHL 1811 has a rather large ratio of $\sim 0.3$. The values for Mrk 231 are very large: 31.5 as observed, and 10.8 corrected for $E(B-V)=0.1$ using an SMC reddening curve (see $\S$ 4). We note that it is not possible to correct C IV from Mrk 231 using the Goobar (2008) reddening curve; as can be seen in Fig. 2, the reddening is completely optically thick at those wavelengths, and the observed continuum and lines have a different origin, perhaps in scattered light (\$7.6). For simulations we set a lower limit of 0.025 on the $\mathrm{He} \mathrm{I}^{*} / \mathrm{C}$ IV ratio, but do not set an upper limit in order to see if we can attain the high values observed from Mrk 231.

\section{Spectral Energy Distributions}

As discussed above, Yan et al. (2015) assumed a spectral energy distribution that maximally favors their interpretation. In this section, we reconstruct their SED, correcting for the observed $\mathrm{X}$-ray emission, and compare it with more typical spectral energy distributions that roughly span the range observed from AGN and quasars. We also consider X-ray weak SED observed from 

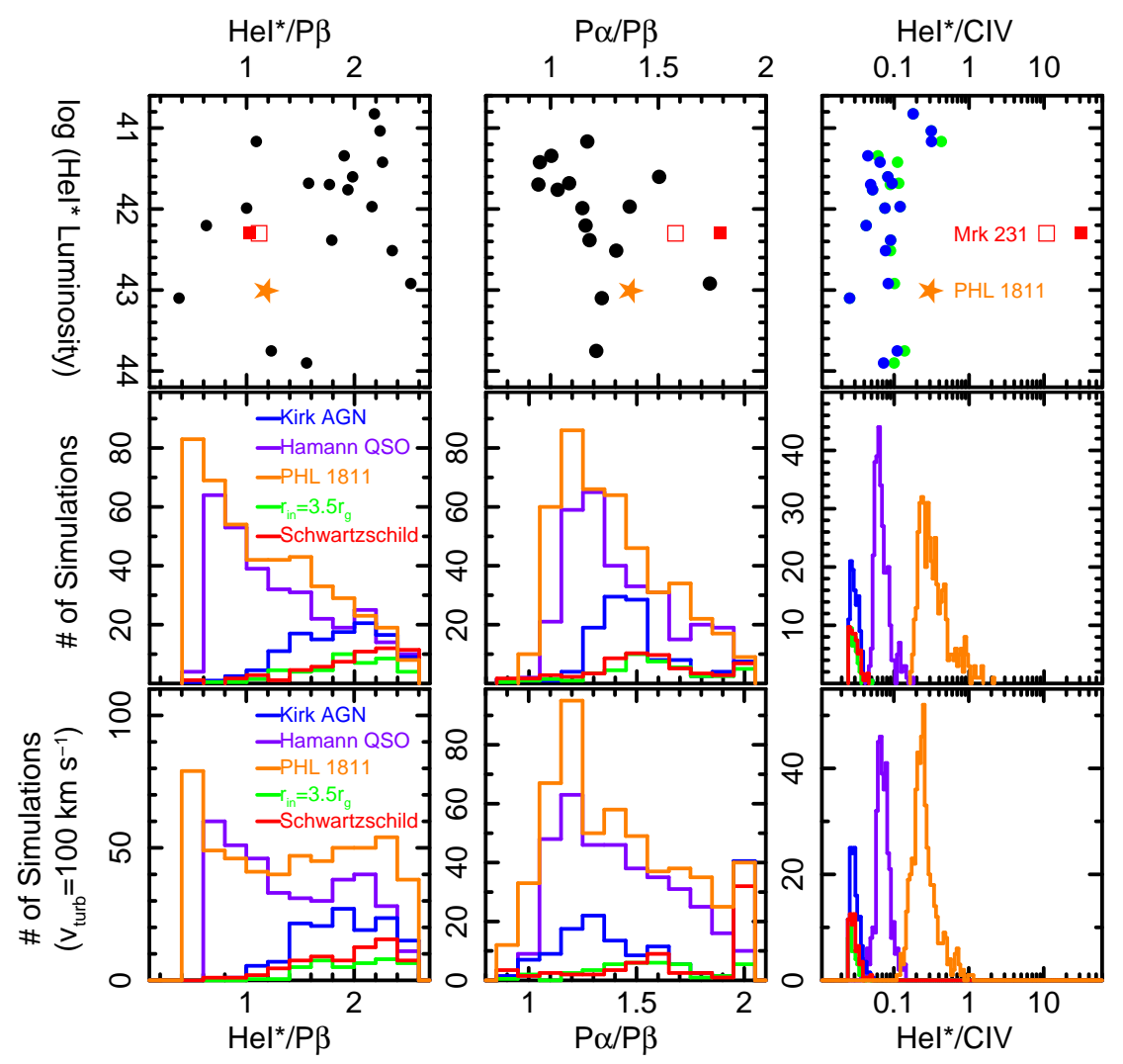

Fig. 3.- Top Panels: Observed He I* $/ \mathrm{P} \beta, \mathrm{P} \alpha / \mathrm{P} \beta$, and $\mathrm{He} \mathrm{I}^{*} / \mathrm{C}$ IV ratios from Mrk 231 (filled red squares), the intrinsically X-ray weak quasar PHL 1811 (orange star), and a comparison sample taken from Landt et al. (2008) (filled circles). Also shown are ratios for Mrk 231 corrected using the reddening curve inferred by Leighly et al. (2014) (for infrared ratios $\mathrm{He}^{*} / \mathrm{P} \beta$ and $\mathrm{P} \alpha / \mathrm{P} \beta$ ) and using an SMC reddening curve with $E(B-V)=0.1$ for the He I*/C IV ratio (open red squares). Mrk 231 and PHL 1811 have typical infrared line ratios, while PHL 1811 has a somewhat high He I*/C IV ratio, due to its weak high-ionization line emission (Leighly et al. 2007b). The He I*/C IV ratio for Mrk 231 is much higher (note the logarithmic $\mathrm{x}$-axis), due to the low C IV flux. It is likely that we do not observed the intrinsic C IV emission from Mrk 231 directly. Middle Panels: The distributions of results from Cloudy simulations found to be consistent with the adopted ranges of $\mathrm{He} \mathrm{I}^{*} / \mathrm{P} \beta$ and $\mathrm{P} \alpha / \mathrm{P} \beta$ ratios, the lower limit of $\mathrm{He} \mathrm{I}^{*} / \mathrm{C}$ IV, and observed values of either He I* flux or equivalent width. These panels show that the Cloudy models are able to match the observed ranges of the comparison sample ratios, and the $\mathrm{He} \mathrm{I}^{*} / \mathrm{P} \beta$ ratio suggests diagnostically useful SED dependence, along with He I*/C IV, assuming appropriate reddening corrections can be made. Bottom Panels: The same as the middle panels, but including a turbulent velocity in the simulations. The results are not substantially different from the static case.

PHL 1811. The properties of the spectral energy distributions are listed in Table 1.

We first considered two spectral energy distributions previously used to model lines from AGN and quasars. A relatively hard one was adopted from Korista et al. (1997) 3 , and a relatively soft one was taken from Hamann et al. (2011)4. These

\footnotetext{
${ }^{3}$ This SED is taken as a typical AGN spectral energy distribution and is called by the Cloudy command AGN kirk, or, equivalently AGN $6.00-1.40-0.50-1.0$.

${ }^{4}$ This SED is called by the Cloudy command AGN
} 
spectral energy distributions are shown in Fig. 4, arbitrarily normalized to intersect the near-UV portion of the SMC-E $(B-V)=0.1$-corrected Mrk 231 spectrum.

Next, we reconstructed the Yan et al. (2015) SEDs. Yan et al. 2015) infer a small amount of intrinsic reddening, between $E(B-V)=0.07$ and $E(B-V)=0.14$, depending on the model, for an SMC reddening curve (Pei 1992). Therefore, we required the spectral energy distributions to intersect the near UV emission observed from Mrk 231 at $2000 \AA$, either as observed, or dereddened by an intermediate value, $E(B-V)=0.1$.

Teng et al. (2014) presented an analysis of Chandra and NuSTAR observations of Mrk 231. They found an absorbed, weak hard X-ray continuum with a flat $(\Gamma \sim 1.4)$ photon index that they take to be the intrinsic X-ray continuum. Yan et al. (2015) did not consider the X-ray emission in their paper. They felt that they were justified in this assumption because Teng et al. (2014) noted that Mrk 231 is X-ray weak $\left(\alpha_{o x} \sim-1.7\right) 5$ However, Teng et al. (2014) inferred that the observed UV is absorbed, and estimated $\alpha_{o x}$ was based their estimate of the intrinsic optical/infrared spectrum (Veilleux et al. 2013, Figure 3), not the observed one. With respect to the observed UV continuum, Mrk 231 is rather X-ray bright.

The deconvolved X-ray spectrum is shown in Figure 5 of Teng et al. (2014). We digitized the "Direct PL" component of that plot between 8.4 and $19.9 \mathrm{keV}$. We multiplied the result by a factor of 1.29 in order to match the $0.5-30 \mathrm{keV}$ luminosity in their Table 1 for the MyTorus model. The Teng et al. (2014) power law is shown in dark gray in Fig. 4. We required that the reconstructed Yan et al. (2015) spectral energy distributions intersect this X-ray data, and have $F_{\nu} \propto \nu^{-0.28}$ (the slope we measured from the digitized spectrum), breaking to a slope of -2 for energies higher than $25 \mathrm{keV}$, the limit of the $N u S T A R$ spectrum.

Yan et al. (2015) assumed an inner radius $r_{i n}=3.5$ gravitational radii $\left(r_{g}\right)$ in order to produce a radiative efficiency of $\eta=0.1$. Their

$\mathrm{T}=200000 \mathrm{~K}, \mathrm{a}(\mathrm{ox})=-1.7, \mathrm{a}(\mathrm{uv})=-0.5, \mathrm{a}(\mathrm{x})=-0.9$.

${ }^{5} \alpha_{o x}$ is the point-to-point slope between the ultraviolet continuum measured at $2500 \AA$ and the X-ray continuum measured at $2 \mathrm{keV}$. best-fitting model yielded $M_{\odot} \sim 4.5 \times 10^{6} \mathrm{M}_{\odot}$ radiating at $0.6 L_{E d d}$ for the smaller-mass black hole. They assumed that the optical-UV spectrum of the smaller black hole is characterized by a sum-of-blackbodies accretion disk model (e.g., Frank et al. 2002). The outer edge of the disk was taken to be 100 times the inner edge. This information was sufficient for us to follow Yan et al. (2015) and compute a disk spectrum from the infrared through the far UV (i.e., through the high-temperature rolloff). The disk spectrum was normalized to the observed $2000 \AA$ flux, and the X-ray spectrum as described above joined to the infrared-through-UV spectrum. This SED is shown in Fig. 4. and information about this continuum is given in Table 1.

When we normalized the $r_{i n}=3.5 r_{g}$ spectrum to the Mrk 231 continuum corrected for $E(B-$ $V)=0.1$ at $2000 \AA$, we found that the emission is super-Eddington $\left(L / L_{E d d}=1.14\right)$. Yan et al. (2015) found it to be sub-Eddington, possibly because they did not include the X-ray emission. So, we normalized the $r_{i n}=3.5 r_{g}$ SED to the observed Mrk 231 continuum at $2000 \AA$, rather than the $E(B-V)$ dereddened one. But in order to give the Yan et al. (2015) model the best chance for success (i.e., the largest possible photoionizing flux), we also considered a Schwartzschild disk, i.e., $r_{i n}=6 r_{s}$, normalized to the Mrk 231 spectrum corrected for intrinsic absorption of $E(B-$ $V)=0.1$; it is also shown in Fig. 4. This SED is not super-Eddington (Table 1).

These two spectral energy distributions have relatively flat values of $\alpha_{o x}$ of -1.28 , when normalized to the observed continuum, and -1.45 when normalized to the $E(B-V)=0.1$ dereddened one. $\alpha_{o x}$ is related to the UV monochromatic luminosity at $2500 \AA$ (e.g., Steffen et al. 2006); those regression relationships predict $\alpha_{o x}$ to be $\sim-1.6$. This is steeper than inferred, but roughly within the regression uncertainty of 0.24 (Steffen et al. 2006, Equation 2). Since, according to the Yan et al. (2015) model, the bulk of the $\mathrm{X}$-ray emission emerges from the central engine of the small-mass black hole, that system should be relatively X-ray bright, like a Seyfert nucleus.

Fig. 2 and Fig. 4 display the intrinsic continuum inferred using the circumstellar reddening model (Leighly et al. 2014). When we used our inferred intrinsic continuum and the extrapola- 
TABLE 1

Properties of Spectral Energy Distributions

\begin{tabular}{|c|c|c|c|c|c|}
\hline \multirow[b]{2}{*}{ Property } & \multicolumn{2}{|c|}{ Comparison Sample } & \multicolumn{2}{|c|}{ Yan et al. 2015} & \multirow{2}{*}{$\begin{array}{c}\text { Possible Intrinsic } \\
\text { PHL } 1811\end{array}$} \\
\hline & Kirk AGN & Hamann QSO & $r_{i n}=3.5 r_{g}$ & Schwartzschild & \\
\hline Reference & Korista et al. (1997) & Hamann et al. (2011) & \multicolumn{2}{|c|}{ Yan et al. (2015)+Teng et al. (2014) } & Leighly et al. (2007a) \\
\hline $\log$ Bolometric $\left[\operatorname{erg~s}^{-1}\right]$ & $\mathrm{N} / \mathrm{A}$ & $\mathrm{N} / \mathrm{A}$ & $44.34^{\mathrm{a}}$ & $44.50^{\mathrm{b}}$ & $45.70^{\mathrm{c}}$ \\
\hline$L / L_{e d d}$ & $\mathrm{~N} / \mathrm{A}$ & $\mathrm{N} / \mathrm{A}$ & $0.39^{\mathrm{d}}$ & $0.56^{\mathrm{d}}$ & $0.17^{\mathrm{e}}$ \\
\hline$\alpha_{o x}$ & -1.40 & -1.70 & -1.28 & -1.42 & -2.26 \\
\hline $\log \mathrm{Q}\left[\right.$ photons $\left.^{-1}\right]$ & $\mathrm{N} / \mathrm{A}$ & $\mathrm{N} / \mathrm{A}$ & 54.36 & 54.61 & 55.61 \\
\hline
\end{tabular}

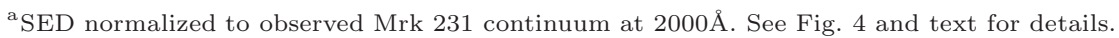

${ }^{\mathrm{b}}$ SED normalized to Mrk 231 continuum corrected for $E(B-V)$ at $2000 \AA$. See Fig. 4 and text for details.

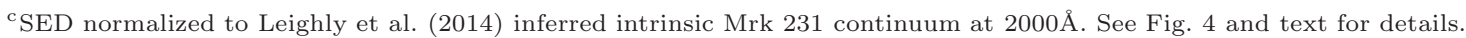

${ }^{\mathrm{d}}$ Calculated using black hole mass $4.5 \times 10^{6} \mathrm{M}_{\odot}$ (Yan et al. 2015).

${ }^{\mathrm{e}}$ Calculated using black hole mass $2.3 \times 10^{8} \mathrm{M}_{\odot}$ (Leighly et al. 2014).

tion of the Teng et al. (2014) power law, we obtained $\alpha_{o x}$ of -2.2 , essentially the same value observed from the intrinsically X-ray weak quasar PHL 1811 (between -2.2 and -2.4 , accounting for X-ray variability, Leighly et al. 2007a). Moreover, Veilleux et al. (2016) also note the similarity between properties of Mrk 231 and PHL 1811 and its analogs. Intrigued by this result, we investigated whether the extreme X-ray weak PHL 1811 continuum could produce the emission lines observed in Mrk 231 in 5.3.

\section{Cloudy Models}

We used the photoionization code Cloudy Ferland et al. 2013) to see if we could produce the $\mathrm{He} \mathrm{I}^{*}, \mathrm{P} \beta, \mathrm{P} \alpha$, and $\mathrm{C}$ IV lines in the range of strengths and ratios observed. We performed a set of simulations for each of the five continua described in 4 . In each case, we perform 5000 or 10,000 simulations with parameters randomly drawn from uniform distributions of ionization parameter $(-3 \leq \log (U) \leq 1.0)$, density $(6 \leq \log (n) \leq 11.5)$, and a combination parameter defined as the difference between the log of the column density $N_{H}$ and the log of the ionization parameter, $\log \left(N_{H}\right)-\log (U)$, that measures the thickness of the gas slab relative to the hydrogen ionization front $\left(22.5 \leq \log \left(N_{H}\right)-\log (U) \leq 24.0\right)$, and has been shown to be useful in analysis of both emission lines and absorption lines Leighly 2004; Casebeer et al. 2006; Leighly et al. 2007b, 2009, 2011, 2014; Lucv et al. 2014). We per- formed the entire set of simulations for a stationary gas, and for a gas with a turbulent velocity $v_{\text {turb }}=100 \mathrm{~km} \mathrm{~s}^{-1}$. A total of 80,000 simulations were performed.

To characterize the strength of the He I* emission, we used either the line flux or the line equivalent width, depending on the circumstance. For the Yan et al. (2015) models, we use the flux of the line, since, as discussed in 3.2 , huge equivalent widths with respect to the photoionizing continuum are required. For the comparison objects, we used the line equivalent width, although comparison of theoretical and observed equivalent widths can be difficult for infrared lines. For example, the Cloudy line fluxes assume full coverage, yet it is known that the broad line region does not fully cover the continuum (or we would always see absorbed continuum spectra in the X-ray band). Leighly (2004) found a covering fraction of 0.05 for intermediate- and low-ionization lines for two rather weak-lined quasars, and we used that value for the lower limit. For the upper limit, we chose a value of 0.5 .

Also, the equivalent width from simulations will be with respect to the AGN continuum, while the observations may include a torus component in the near infrared, and/or host galaxy. The comparison sample is dominated by nearby AGN and quasars, and it is likely that the spectroscopic slit excluded much of the galaxy contribution. The He I* line, at $10830 \AA$, occurs just at the 1 -micron break, where the accretion disk continuum and the torus contribution are approximately equal, and so 


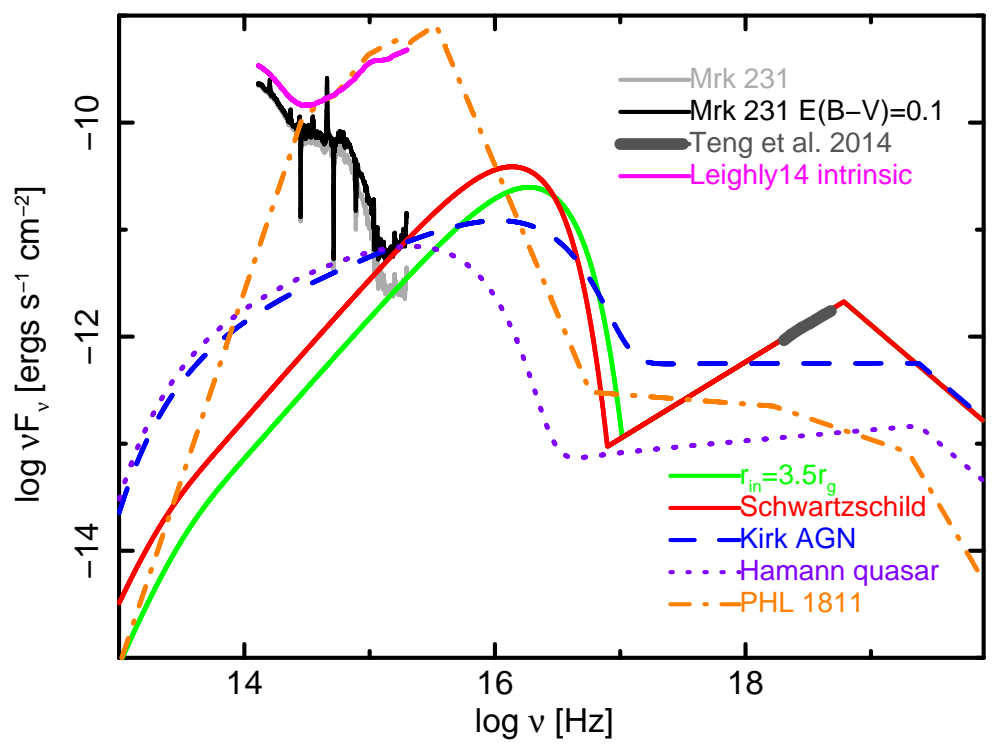

Fig. 4.- Continua used for Cloudy simulations, overlaid on the merged Mrk 231 near-UV-to-near-IR merged spectrum from Leighly et al. (2014), and the X-ray results from Teng et al. (2014). Representative "Kirk AGN" and "Hamann quasar" SEDs were used to demonstrate that the Cloudy simulations can produce observed He I* equivalent widths, and $\mathrm{He} \mathrm{I}^{*} / \mathrm{P} \beta, \mathrm{P} \alpha / \mathrm{P} \beta$, and $\mathrm{He} \mathrm{I}^{*} / \mathrm{C}$ IV ratios in the comparison sample (5.1). The " $r_{i n}=3.5 r_{g}$ " and "Schwartzschild" continua were used to simulate the Yan et al. (2015) model (\$5.2). Finally, the "PHL 1811" continuum was used to investigate the line fluxes and ratios for an X-ray weak continuum (\$5.3).

it is expected that the torus contribution will not dominate.

In addition, the near-infrared portion of the continuum, near 1 micron, lies far from the hydrogen continuum shortward of $911 \AA$, which sets the level of the photoionizing flux. For example, the two SEDs used in 5.1 have optical-UV spectra with $F_{\nu} \propto \nu^{-0.5}$, typical of quasars and AGN (e.g., Natali et al. 1998). But the intrinsic slope is not uniform among AGN, and the larger the difference from $\nu^{-0.5}$, the larger the incurred uncertainty in the equivalent width in the infrared band.

We addressed these concerns by considering a large range of equivalent width for He I*. The observed equivalent width range in the comparison sample is $30-300 \AA$, and therefore we accept simulations producing equivalent widths for full covering between $60 \AA$ (i.e., lower limit on equivalent width divided by upper limit on covering fraction) and $6000 \AA$ (i.e., upper limit on equivalent width divided by lower limit on covering fraction).
Generally speaking, we chose very generous bounds on every parameter in order to give the Yan et al. (2015) model the best chance. Therefore, when we show it is not feasible, our result cannot be attributed to an artificial or arbitrary limitation to the considered range of parameter space.

\subsection{Cloudy Models of the Comparison Sample}

We first needed to establish that Cloudy can explain the observed He I* intensity and the observed line ratios from typical objects to be confident that we could use the Cloudy results to analyze special cases. We bracket the plausible range of SED shapes by considering a hard, X-ray bright one from (Korista et al. 1997) that may be appropriate for Seyfert galaxies, and a soft one that may be appropriate for QSOs (Hamann et al. 2011). We extracted the predicted He $\mathrm{I}^{*}, \mathrm{P} \beta, \mathrm{P} \alpha$, and $\mathrm{C}$ IV fluxes from the simulation results using these 
two SEDs, and computed the $\mathrm{He} \mathrm{I}^{*} / \mathrm{P} \beta, \mathrm{P} \alpha / \mathrm{P} \beta$, and He I*/C IV ratios.

We accepted solutions that were consistent with the line ratios in the ranges discussed in 3.4 He I* $/ \mathrm{P} \beta$ between 0.1 and $2.5, \mathrm{P} \alpha / \mathrm{P} \beta$ between 0.9 and 2.0 , and $\mathrm{He} \mathrm{I}^{*} / \mathrm{C}$ IV larger than 0.025 . For the He I* flux constraint, we used the equivalent width range (for full covering) between 60 and $6000 \AA$, as discussed in $\$ 5$,

We show the histograms of results from accepted simulations in the lower panels of Fig. 3 Interestingly, SED dependence is present in all the ratios, to a greater or lesser degree. The soft continuum leans towards smaller values of $\mathrm{He} \mathrm{I}^{*} / \mathrm{P} \beta$, while the hard continuum yields a larger value of this ratio. This is plausibly a consequence of the the stronger helium continuum in the hard SED. Both spectral energy distributions favor an intermediate value of $\mathrm{P} \alpha / \mathrm{P} \beta$, interestingly close to the mean value observed. This ratio depends principally on optical depth, so a great deal of SED dependence is not expected.

The two SEDs produced the largest differences in the $\mathrm{He} \mathrm{I}^{*} / \mathrm{C}$ IV ratio. The hard SED produces low values of this ratio; that is, it yields relatively large C IV fluxes. This is expected; a harder SED will produce a hotter photoionized gas (e.g., Leighly et al. 2007b, Fig. 14), and C IV is an important coolant, so the proportion of C IV compared with a recombination line like He $\mathrm{I}^{*}$ can be expected to be large when the SED is hard. The softer SED, yields a lower value and matches the observed distribution of He I*/C IV ratios nicely.

Fig. 5 shows the Cloudy input parameters for the accepted simulations. The softer SED tends to favor a higher ionization parameter than the harder one. This is expected; a higher photon flux is necessary for a soft SED to produce the required He I* flux or equivalent width.

There is a strong localization in densities favored due to opacity of the Paschen lines. The simulation results show that for fixed $\log U$ and $\log N_{H}-\log U$, both $\mathrm{P} \alpha$ and $\mathrm{P} \beta$ became thermalized at larger densities (i.e., the increase of line flux with density broke to a flatter slope), but with $\mathrm{P} \alpha$ becoming thermalized at slightly lower densities than $\mathrm{P} \beta$, resulting in a decrease in the $\mathrm{P} \alpha / \mathrm{P} \beta$ ratio to less than the observed upper limit of $\sim 2$ near $\log n_{e}=7.5$, and thus providing a constraint on the density on the low end. At the same time, $\mathrm{P} \beta$ became thermalized much faster than He I*, resulting in a ratio higher than the observed upper limit for the He I* $/ \mathrm{P} \beta$ ratio of $\sim 2.5$ for values greater than $\log n_{e}=9.5$ and thus constraining the density on the high end.

To investigate the influence of optical depth, we ran the simulations including a turbulent velocity $v_{\text {turb }}=100 \mathrm{~km} \mathrm{~s}^{-1}$. The effect of turbulence is to decrease optical depths (e.g., Bottorff et al. 2000). The chosen value of $v_{\text {turb }}$ is much larger than the thermal line width in a photoionized gas (about $15 \mathrm{~km} \mathrm{~s}^{-1}$ ). Physically, it may represent actual macro-turbulence, or differential velocity. This value was chosen arbitrarily because it was large enough to show an effect (no significant effect was observed for $v_{\text {turb }}=15 \mathrm{~km} \mathrm{~s}^{-1}$ ), and a single value allowed us to understand the effect of turbulence qualitatively. The principal effect of turbulence was a shift of the favored range of density. As expected, the opacity is lower in the turbulent case, and thermalization becomes important at higher densities than in the stationary case.

There were a greater number of simulations accepted for larger values of $\log \left(N_{H}\right)-\log (U)$, i.e., column density, as expected, since the Paschen lines are produced predominantly in the partially ionized zone, located beyond the hydrogen ionization front, i.e., $\log \left(N_{H}\right)-\log (U) \gtrsim 23.2$.

These simulations show that these typical AGN and quasar SEDs are able to produce the He I* equivalent widths, and $\mathrm{He} \mathrm{I}{ }^{*} / \mathrm{P} \beta, \mathrm{P} \alpha / \mathrm{P} \beta$, and $\mathrm{He} \mathrm{I}^{*} / \mathrm{C}$ IV ratios observed from the typical objects in the comparison sample. We now turn to the more specialized cases.

\subsection{Cloudy Models using the Yan et al. (2015) Spectral Energy Distributions}

Cloudy models employing the $r_{i n}=3.5 r_{g}$ and the Schwartzschild spectral energy distributions were used to evaluate the viability of the Yan et al. (2015) model. We first asked a basic question: can these SEDs, normalized as described to the observed UV and X-ray emission, produce the He I* flux observed, given the range of observed He I* $/ \mathrm{P} \beta$ ratios? The advantage of this minimal set of constraints is that it uses only infrared spectral data, and lines that are close to one an- 


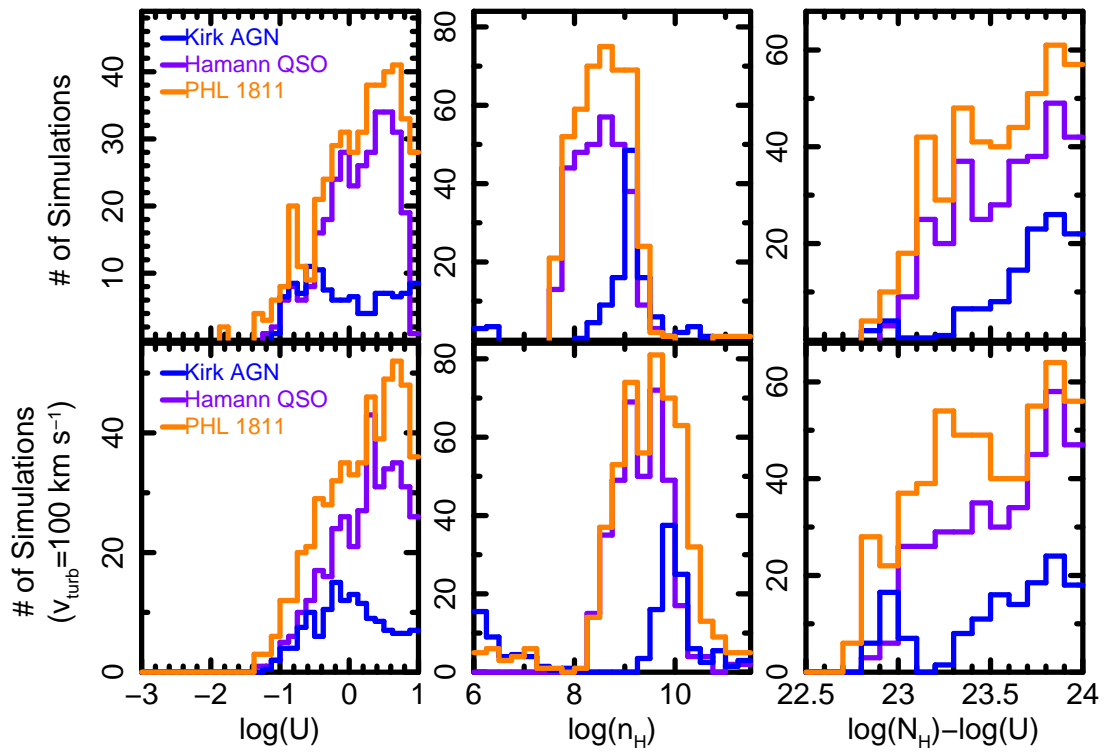

Fig. 5.- The distributions of input parameters from Cloudy simulations in which the results were selected to be consistent with the observed ranges of $\mathrm{He} \mathrm{I}^{*} / \mathrm{P} \beta$ and $\mathrm{P} \alpha / \mathrm{P} \beta$ ratios, and the lower limit of He I*/C IV ratios in the comparison sample, and either He I* flux or equivalent width; see text for details. We found that the simulations are consistent with a rather high ionization parameter (higher for softer SEDs, as expected, to meet the requirement of a sufficiently strong helium continuum to yield the observed He I* emission), an intermediate range of density, and a relatively high column density, i.e., $\log \left(N_{H}\right)-\log (U) \geq \sim 23$. The top panels show the results for the stationary case, and the bottom panels show the results for $v_{\text {turb }}=100 \mathrm{~km} \mathrm{~s}^{-1}$. The principal difference is the favored density range, which is shifted to higher densities for the turbulent case because of reduced thermalization of the Paschen lines as a consequence of lower opacity.

other, and so will be minimally impacted by reddening regardless of model. We found that neither of these two SEDs could produce the observed He $I^{*}$ flux when constrained to produce the observed range He $I^{*} / P \beta$ ratios, even for a covering fraction of the broad line region equal to 1 . This result is not unexpected given the discussion in $\S 3.2$ i.e., that the near-IR equivalent widths would have to be $\sim 100$ larger than normal with respect to the photoionizing continuum in order to explain the near-infrared line emission observed. It is simply not reasonable to expect the continuum from a $4.5 \times 10^{6} \mathrm{M}_{\odot}$ black hole to be able to power the broad line region emission of a $>10^{8} \mathrm{M}_{\odot}$ quasar.

As noted in $\S 2$, Yan et al. (2015) state, in their $\S 6$, that there are sufficient photons in the smallblack-hole-mass continua to explain the $\mathrm{H} \beta$ emission. We believe that they underestimated the required photon flux by using $\mathrm{H} \beta$, since the spec- trum is significantly redddened in that region. $\mathrm{H} \beta$ is strongly blended with the strong Fe II, making it difficult to measure, but the spectral fit shown in Leighly et al. (2014) Fig. 6 yielded an estimate of the observed flux in $\mathrm{H} \beta$ of $2.8 \times 10^{-13} \mathrm{erg} \mathrm{s}^{-1} \AA^{-1}$, with the dereddened value being 5.4 times larger. The observed photon flux in $\mathrm{H} \beta$ is then $2.6 \times$

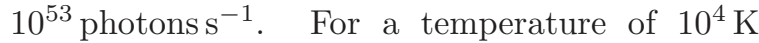
and a density of $10^{6} \mathrm{~cm}^{-3}$, and assuming Case B (Osterbrock \& Ferland 2006), we find that the photonionizing photon flux must be a factor of $\alpha_{B} / \alpha_{P \alpha}^{e f f}=8.44$ times larger than the photon flux in the line, i.e., $Q=2.2 \times 10^{54}$ photons s $^{-1}$. This calculation assumes that the covering fraction is $100 \%$; for a more realistic covering fraction, the photon flux would have to be even larger. The photoionizing fluxes for the Yan et al. (2015) continua are given in Table1. The photoionizing flux from the $3.5 r_{g}$ continuum just matches the re- 
quired value (so full coverage is necessary), and the value from the Schwartzschild continuum exceeds the required value by a factor of 1.8 , requiring a covering fraction of $\sim 50 \%$. However, the dereddened $\mathrm{H} \beta$ requires $Q=1.2 \times 10^{55}$ photons $^{-1}$, exceeding the Yan et al. (2015) ionizing photon fluxes by factors of 3-5.

$\mathrm{P} \alpha$ is subject to less reddening, and may provide a more accurate estimate of the required photoionizing flux. The flux of $\mathrm{P} \alpha$ was measured to be $8.1 \times 10^{-13} \mathrm{erg} \mathrm{s}^{-1} \mathrm{~cm}^{-2}$, for a photon flux of $2.9 \times 10^{54}$ photons ${ }^{-1}$ in $\mathrm{P} \alpha$. The $\alpha_{B} / \alpha_{P \alpha}^{e f f}=6.99$ for this line implies that $Q=2.0 \times 10^{55}$ photons s $^{-1}$ for full coverage. This value exceeds the photoionizing flux from the Yan et al. continua by a factor of 5-9. These values are roughly consistent with the above estimate from the unreddened $\mathrm{H} \beta$ above. Thus, we conclude that Yan et al. 2015) underestimated the required photon flux because they used the significantly reddened $\mathrm{H} \beta$ line.

Moreover, while adequate for planetary nebula and $\mathrm{H}$ II regions, the nebular approximation is well known not to be appropriate for AGN (e.g., Davidson \& Netzer 1979), where the bulk of the hydrogen line emission arises from the partially ionized zone. Our more complete treatment uses infrared lines that are less likely to be affected by reddening, and requires that we only consider the simulations that yield $\mathrm{He} \mathrm{I}^{*} / \mathrm{P} \beta$ ratios within the range observed from AGN. Fig. [5] shows that most of the one-zone models that meet this requirement lie in the partially ionized zone (with values of $\log N_{h}-\log U>\sim 23.2$ ), as expected.

We were also interested in whether these SEDs could explain the unusually large He I*/C IV ratio observed. The equivalent width uncertainty outlined in $\$ 5$ is exacerbated by the fact that the sum-of-blackbodies accretion disk spectrum has a long wavelength spectrum described by $F_{\nu} \propto$ $\nu^{1 / 3}$. Such a steep spectrum is not generally seen in AGN; this is one of the problems hampering our understanding of accretion disks (e.g., Koratkar \& Blaes 1999). Typical values of the optical to UV slope are observed to be around -0.5 (e.g., Natali et al. 1998; Vanden Berk et al. 2001) to -0.3 (e.g., Francis et al. 1991; Selsing et al. 2015). For SEDs with the same value of $F_{\lambda}$ at $911 \AA$, the continuum will be 7.87 times weaker at $10830 \AA$ for a $F_{\nu} \propto \nu^{1 / 3}$ continuum than for a $F_{\nu} \propto \nu^{-0.5}$ continuum. To compensate for this additional uncertainty, we increase the upper limit on the allowed equivalent widths by a factor of 7.87 .

The results are shown in Fig. 3. The $r_{i n}=3.5 r_{g}$ and Schwartzschild SEDs produce results that are similar to those obtained with the harder SED explored in $\$ 5.1$. This is not surprising given the large fraction of photoionizing flux in the extreme UV and the flat values of $\alpha_{o x}$. Specifically, they predicted only very low values of $\mathrm{He} \mathrm{I}^{*} / \mathrm{C}$ IV, and cannot explain the high value observed in Mrk 231.

\subsection{Cloudy Models using the PHL 1811 Continuum}

As discussed in $\S$, the PHL 1811 continuum may be similar to the intrinsic continuum in Mrk 231. In this section, we explore whether it can produce the observed emission lines. We normalized the SED to the Leighly et al. (2014) intrinsic continuum, and required the simulations to produce the observed He $\mathrm{I}^{*}$ flux. We first deredden the He I* flux using the inferred circumstellar reddening curve from Leighly et al. (2014). A large fraction of the simulations are able to meet these selection criteria, and their properties are shown in Fig. 3 and Fig. 5 .

Fig. 3 shows that the $\mathrm{He} \mathrm{I}^{*} / \mathrm{P} \beta$, and $\mathrm{P} \alpha / \mathrm{P} \beta$ ratios strongly resemble those produced by the soft SED considered in $\S 5.1$ but the He I*/C IV ratio is higher than for the other SEDs, and is consistent with the observed value from PHL 1811. This is not surprising as the very X-ray weak PHL 1811 SED has been shown to produce weak high-ionization lines (Leighly et al. 2007b). Yet the $\mathrm{He} \mathrm{I}^{*} / \mathrm{C}$ IV ratio does not approach the very high value exhibited by Mrk 231. As PHL 1811 is intrinsically exceptionally X-ray weak (Leighly et al. 2007a), with exceptionally small C IV equivalent width (Leighly et al. 2007b), we suspect that it would be difficult to produce a much higher ratio intrinsically. This is evidence that the He I*/C IV ratio in Mrk 231 is high because of reddening and is not intrinsic. The UV continuum and broad-line region in Mrk 231 are likely not seen directly at all.

\section{Evidence for Resolved Far UV Emission}

If the weak far-UV emission is not the continuum from the smaller-black hole in a milli-parsec 
black hole binary system, then what is it? In this section, we report the discovery of evidence for resolved FUV emission in the archival HST STIS data of Mrk 231 that is supported by analysis of an HST FOC image. This result suggests that the far-UV continuum does not originate in the central engine.

\subsection{Spatial Analysis of the HST STIS Ob- servation}

Leighly et al. (2014) reported analysis of the APO TripleSpec observation of Mrk 231. We found that the spectral trace was broader in the He I*10830 trough than it was at unabsorbed wavelengths. This result indicated that the trough is partially filled in by extended emission. Given the relatively poor spatial resolution available in the ground-based observation, and the infrared bandpass, the extended emission is probably the host galaxy.

The observed-frame HST STIS spectrum is shown in the lower panel of Fig 6 (see also Veilleux et al. 2016, Fig. 3). The Mg II trough observed near $2870 \AA$, and the low-excitation Fe II troughs near $2450 \AA$ and $2674 \AA$ (observed wavelengths) are approximately flat and have approximately the same flux level as the far UV continuum. This suggests that those troughs are saturated and suffer partial covering, and the far UV continuum partially fills in the troughs. It is therefore conceivable that interesting constraints on the origin of the far-UV emission might be obtained from performing the same type of analysis on the STIS data as was performed on the APO TripleSpec data.

Analysis of the spatial profile of the STIS is not trivial. For example, the detector under samples the point spread function; the STIS line spread function for the G230L detector at $2400 \AA 6$ has a FWHM of 1.67 pixels. As noted in the STIS Data Handbook7, this property affects the rectified 2D images. Specifically, the spectra extracted from a single row of the rectified $2 \mathrm{D}$ images produced by the standard pipeline processing include artifacts (scalloping) due to interpolation from one row to the next. Improved rectification can be achieved by using wx2d, a program available in the IRAF package STSDAS. This program employs a wavelet interpolation for improved rectification (Barrett \& Dressel 2006). We used this program on the four STIS G230L unrectified images, and then added them together. For comparison, one observation of the bright $z=0.192$ quasar PHL 1811 was analyzed in the same way.

Spatial profiles were constructed for each wavelength bin between 1950 and $3127 \AA$ (observed frame, to facilitate comparison with PHL 1811). The signal-to-noise ratio for a single wavelength bin was low, so the median among the central wavelength bin and a set number of wavelength bins to each side was used. The signal-to-noise ratio was poorest at shorter wavelengths, so the number of bins on each side was chosen to be 10 and 5 for wavelengths shorter and longer than $2600 \AA$ respectively. The nearly-negligible background was estimated to be the median of the 10 pixels on each side of the central 13 pixels. The central 13 pixels of the background-subtracted profile were oversampled by a factor of 10 , and the cumulative histogram was compiled and normalized. Finally, the pixel locations of the 0.25 , 0.5 , and 0.75 levels of the cumulative histogram were identified by interpolation. The robustness of this procedure was confirmed by varying various parameters in the analysis, including the size of the central region, the size of the background region, and the number of pixels used to construct a profile.

The results are shown in Fig. 6, left side. The top panel shows the distance between the pixel location of the $0.25,0.5$, and 0.75 levels of the cumulative histogram and the fitted center of the mean profile of the whole image. Superimposed are results obtained by performing the same analysis on the spatial profiles of the bright quasar PHL 1811, and on the STIS line spread function profile for the G230L grating at $2400 \AA$ using a 0.2 arcsecond slit. PHL 1811 is a bright, unresolved point source, so ideally, the pixel locations of the $0.25,0.5$, and 0.75 levels should coincide with those from the STIS line spread function. The correspondence is good at long wavelengths, but less so at shorter wavelengths, where the 0.25 level location for PHL 1811 sags below the STIS line spread function result by $\sim 0.2$ pixel. This

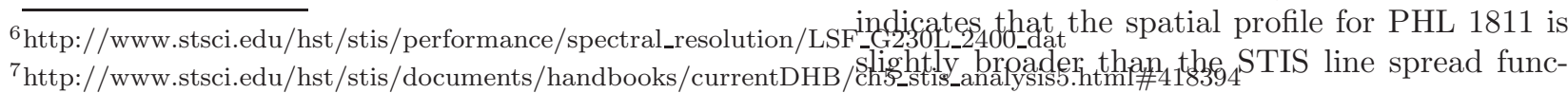

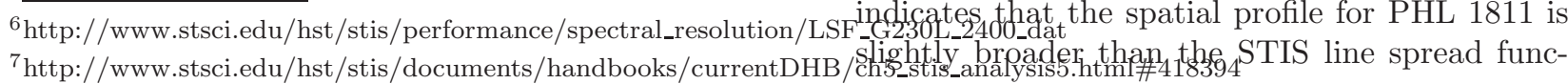



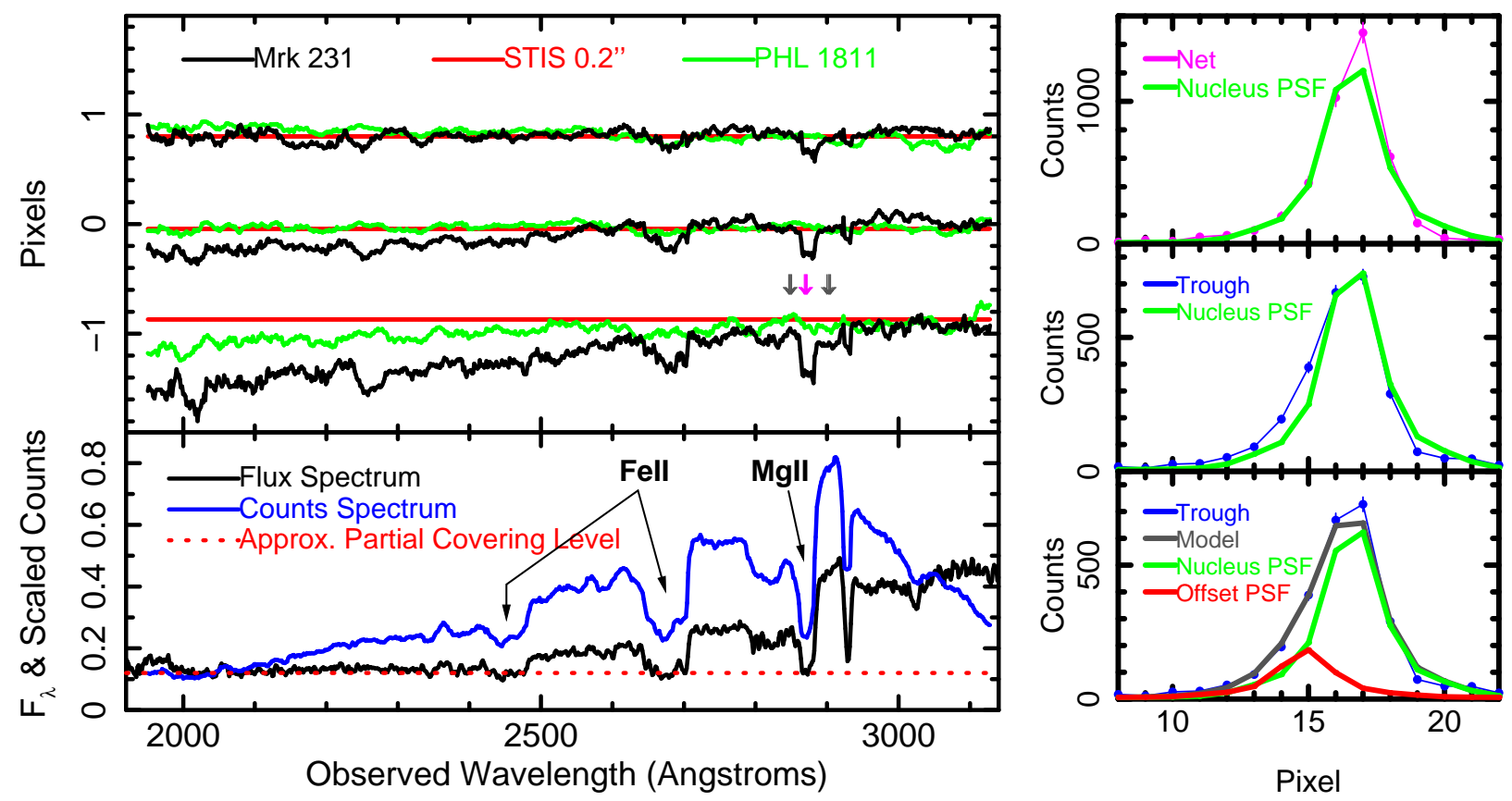

Fig. 6.- Analysis of the spatial profiles of the HST STIS G230L observation of Mrk 231 indicating evidence for resolved emission in the far UV. Left: The lower panel shows the flux and counts spectrum. An approximate constant extrapolation of the far UV continuum to longer wavelengths (red dashed line) suggests that the Fe II and Mg II troughs are saturated and filled in by the same continuum. The upper panel shows the analysis of the spatial profiles. The $0.25,0.5$, and 0.75 levels (bottom, middle, and top traces) of the normalized cumulative histogram are shown for the STIS G230L point spread function for a 0.2 arcsecond slit at $2400 \AA$ (red line), the comparison point source PHL 1811 (green line), and Mrk 231 (black line). An offset of the 0.25 level is seen for Mrk 231, indicating extended emission, especially at short wavelengths, and in the Mg II trough near $2873 \AA$ and Fe II around $2680 \AA$. Right: Analysis of profiles accumulated near the magenta arrow in the left panel (the trough) and the difference between the mean of the gray arrows and the trough (the net). The net profile is fit reasonably well by the STIS PSF (top), but the trough exhibits significant left-side residuals when fit with the same PSF (middle). A good fit is obtained with an additional component offset by $1.72 \pm 0.12$ pixels, corresponding to $36.5 \pm 2.5 \mathrm{pc}$.

tion. Part of the difference could be due to the fact that at shorter wavelengths the STIS line spread function is broader with more prominent wings. The remaining difference is taken to represent the residual systematic uncertainty in the rectification of the $2 \mathrm{D}$ images.

The Mrk 231 profile differs from both the STIS line spread function and the PHL 1811 profile. While the pixel location of the 0.75 cumulative histogram level coincides with that of the STIS line spread function and the PHL 1811, the pixel locations of the 0.5 and especially the $0.25 \mathrm{lev}-$ els are offset. This indicates that the spatial pro- file of Mrk 231 is quite a bit broader than that expected from a point source, especially at short wavelengths, as well as being asymmetric. The count rate is low at short wavelengths, so one might be tempted attribute this offset as systematic uncertainty in the in the rectification of the $2 \mathrm{D}$ image. However, the asymmetry is also observed in the absorption line troughs. This is most clearly seen in the Mg II trough observed around $2873 \AA$, and the low-excitation Fe II trough observed around $2680 \AA$, but is also detectable to a lesser extent in the $\mathrm{Mg}$ I trough observed around $2930 \AA$. This is precisely the behavior expected if 
the far-UV continuum is extended and fills in the bottoms of saturated troughs.

What is the physical extent of the asymmetry? We compiled profiles characterizing the Mg II trough, centered around $2870 \AA$, and marked on Fig. 6] by a magenta arrow, and total NUV continuum emission, taken to be the mean of the profiles centered around 2848 and $2902 \AA$, marked on Fig. 6 by gray arrows. (We used the mean of two points bracketing the $\mathrm{Mg}$ II trough to approximately compensate for the monotonic decrease of the grating effective area at these wavelengths). At each point, we constructed the profile using the sum of the central pixel and 5 pixels on each side to increase the signal-to-noise ratio. As the STIS MAMA is a photon counting detector, we assume that the uncertainty on these counts profiles is Poisson.

If the Mg II trough is filled in by the far UV continuum, the difference between the total continuum and the trough continuum profiles, referred to as the net continuum profile, can be assumed to characterize the profile of the NUV point source, in particular, its spatial location. We fit this profile using CIAO Sherpa 8 with a template model created from the STIS G230L line spread function a range of pixel offsets. to determine the pixel location of the nucleus. The best fit to the net profile locates the pixel location in the spatial direction of the nucleus on the 2-D spectral image (top right panel of Fig. 6).

If Mrk 231 were consistent with a point source at every wavelength, then spatial profiles compiled at any wavelength should be well fit by the STIS G230L spatial profile with the same offset as the net profile. The second panel in Fig. 6] shows the trough profile subject to such a fit. A significant wing is observed on the left side of the profile, invalidating the assumption that Mrk 231 FUV emission is consistent with the nuclear point source, and indicating the presence of extended emission.

In order to quantify the extent of the extended emission, we make the simple assumption that the Mrk 231 FUV profile consists of the nuclear emission plus another, offset component. We fit the trough profile with the net profile model component, and a second offset profile. The result is

\footnotetext{
${ }^{8}$ http://cxc.harvard.edu/sherpa4.8/
}

shown in the lowest right panel of Fig. 6. This model provides a good fit to the wing on the left side of the profile. The offset component accounts for $20 \%$ of the total flux, and the separation is $1.73 \pm 0.12$ pixels, corresponding to $0.0424 \pm 0.0029$ arcseconds. At the distance of Mrk 231, 1 arcsecond corresponds to 863 parsecs (Veilleux et al. 2013), so the offset is $36.5 \pm 2.5 \mathrm{pc}$. We note that this is a lower limit on the offset, since the slit width was 0.2 arcseconds (i.e., 8.16 pixels) and we don't know the relative orientation of the slit and the offset emission.

\subsection{Spatial Analysis of the $H S T$ FOC F210M Image}

The analysis presented in 6.1 suggests the presence of far-UV resolved emission on the scale of 0.042 arcseconds. The diffraction-limited resolution for $H S T$ at $2000 \AA$ is 0.017 arcseconds, so in principle the resolved emission could be detected in an image. The archive contains only one imaging observation in the far UV, a 596.5second observation taken 1998 Nov 28 using the Faint Object Camera (FOC) with the F210M filter (pivot wavelength $=2180 \AA, 162 \AA$ RMS bandwidth) as part of the imaging polarimetry campaign on Mrk 231. The plate scale for the $\mathrm{f} / 96$ relay is $0.01435 \pm 0.00007$ " per FOC pixel. The polarimetry observations were made using F346M, with central wavelength $3400 \AA$, and are therefore dominated by the unresolved near-UV/optical component, and so can't be used to search for extended emission. The results of the imaging polarimetry were reported in Gallagher et al. (2005), but the far UV image, which was made with only F210M filter and no polarimetry elements, was not discussed in that paper.

Gallagher et al. (2005) describe some of the difficulties in identifying extended emission very close to the point source in an FOC image. Each filter has its own distinct point-spread function. For example, examination of Table 9 in the FOC Instrument Handbook 9 shows that the 210M filter has particularly extended wings compared with some of the optical filters, with $\sim 20 \%$ of the flux beyond $\sim 8$ pixels. To account for this complication, we analyzed the FOC PSF image for the F210M

\footnotetext{
${ }^{9}$ http://www.stsci.edu/hst/foc/documents/handbooks/foc_handbook.html
} 
filter 10 for comparison. But according to the FOC Instrument Handbook, there are additional potential problems. For example, there can be geometrical distortion. Usually well calibrated using the reseau marks in the larger format $(512 \times 512)$ observations, there may not be sufficient information for that calibration in the smaller formats; the Mrk 231 observation was made in the $128 \times 128$ format. Moreover, there is variation in the distortion during the detector warmup period, and while no data is taken during the initial warmup period, residual variation on the order of $0.25 \%$ in the plate scale can be present during the next two hours. According to the timeline11, this was the second observation in the sequence. We can't compare with the polarimetry observations, as the polarimetry imaging elements add their own PSF. On the other hand, it is not clear that geometric distortion would be an important effect on the small scales that we are interested in. Finally, there are also known to be small changes in telescope focus due to "breathing" 12 .

We analyzed the Mrk 231 observation and the PSF observation in parallel using the CXC Ciao Sherpa package. We first fit the two images with co-axial two-dimensional Gaussian profiles plus a background. We found that in each case, four Gaussians were sufficient to empirically describe the PSF. The radial profiles from these fits are shown in Fig. 7. The rather broad base corresponds to the particularly extended wings known to characterize this filter.

The third panel of Fig. 7 shows the empiricallyfit radial profiles for Mrk 231 and the PSF observation, with the constant background subtracted, normalized, and overlaid on the Mrk 231 radial profile. Mrk 231 shows significant excess on the 1-4 pixel scale.

Sherpa offers the capability of fitting the image with a 2-dimensional model after convolving with a PSF image. We fit the Mrk 231 observation with a 2-D Gaussian and a constant, using the background-subtracted PSF observation image as the PSF. If the Mrk 231 image were consistent with the detector PSF, the width of the Gaussian would be equal to zero. Instead, we find a best-

10 http://www.stsci.edu/hst/foc/calibration/f96_costar.html

${ }^{11}$ http://www.stsci.edu/ftp/observing/weekly_timeline/1998_timelinesttingeling_11_22_9\$ $\lambda \lambda 1548,1551$ was used to probe ${ }^{12}$ http://www.stsci.edu/hst/foc/documents/abstracts/foc_isr9801.pdf the ultraviolet, to see whether that emission line fit value of $3.42 \pm 0.11$ pixels, corresponding to $0.0491 \pm 0.0016$ arcsecond, or $42 \mathrm{pc}$. The fit is good, and no distinguishable improvement in fit is obtained by adding another Gaussian. The radial profile of the Gaussian model convolved with the PSF is shown in the bottom panel of Fig. 7.

The FOC PSF is known to not be azimuthally symmetric; moreover the PSF observation places the PSF star near a reseau mark. So we also fit the Mrk 231 image using a PSF developed from the Gaussian-fit model of the PSF image. The results were essentially the same; the width of a single 2D Gaussian was $3.35 \pm 0.12$ pixels, corresponding to $0.0481 \pm 0.0017$ arcsecond, or $41 \mathrm{pc}$.

Beyond the extended emission, the FOC image provided no exceptional indication of structure. For example, a 2-D elliptical model does not provide notable improvement in fit. But the signal-to-noise ratio of this image is low. The peak pixel contained 93 photons, and we estimate only $\sim 1600$ source photons for the image within a radius of 5 pixels, for an average of 20 photons per pixel. The STIS spatial analysis suggested that if the offset component were modeled as a point source, its intensity would be $20 \%$ of the main component. Simulated images show that such an offset point source might be just detectable in an image with these statistics. But the emission might be truly extended, in which case an asymmetry, lying in the wings of the nuclear PSF, would be very difficult to detect in an image with these statistics.

\section{Discussion}

\subsection{Summary}

We used observed emission lines from Mrk 231 and a comparison sample to investigate the binary-black hole model proposed by Yan et al. (2015), and to compare it with the circumstellar absorption model proposed by Leighly et al. (2014). We used infrared lines, which are subject to minimal reddening regardless of the model. The He $I^{*} \lambda 10830$ line yielded information about the ionization parameter, while the $\mathrm{P} \beta$ line at $12818 \AA$ and the $\mathrm{P} \alpha$ line at $18751 \AA$ yielded information about the density (due to thermalization at high density) and column density of the emit- 


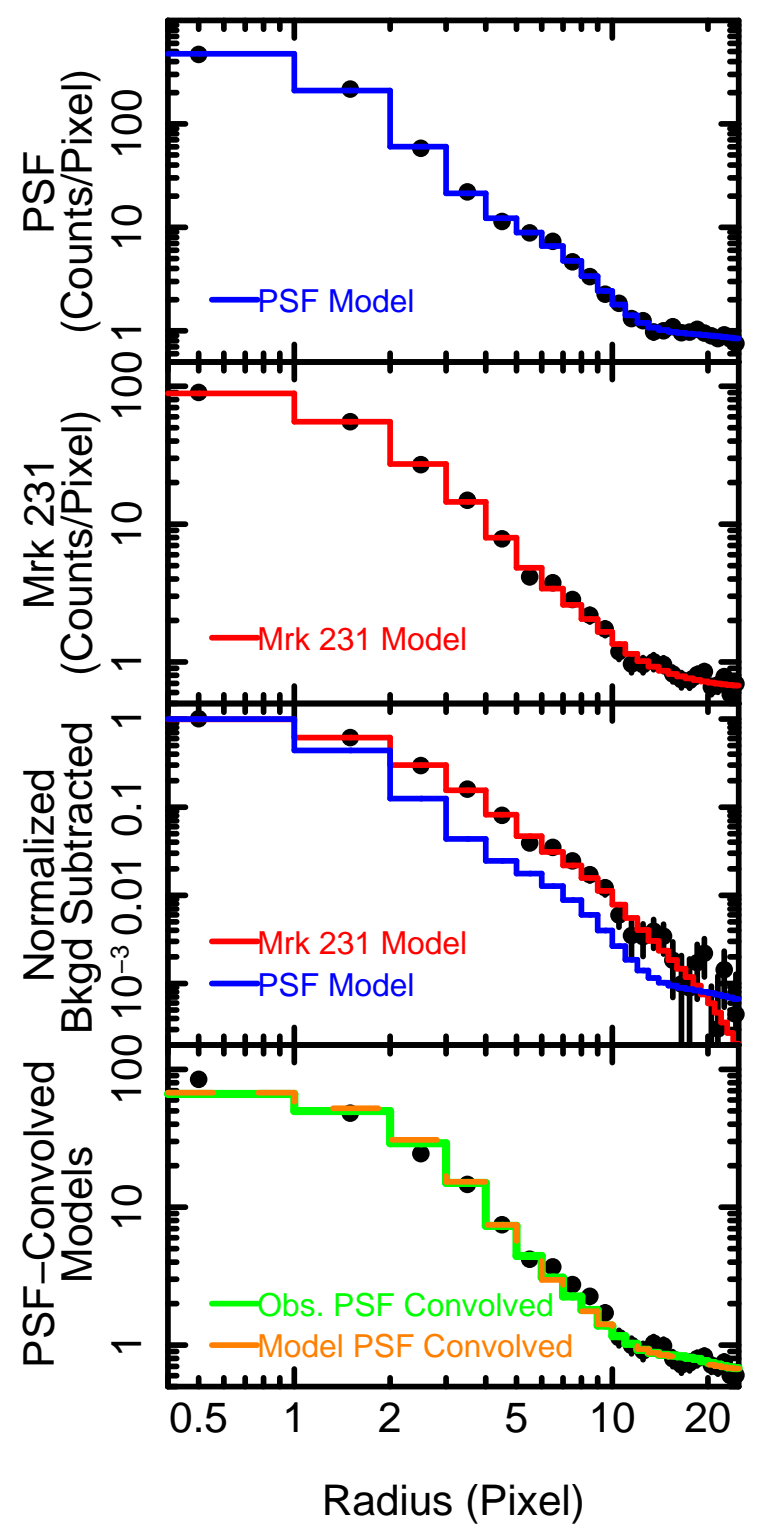

Fig. 7.- Analysis of the HST FOC F210M observation of Mrk 231. The top panel shows the radial profile of the FOC F210M PSF image, fitted with an empirical model consisting of a constant background plus four co-aligned 2-D Gaussian models. The second panel shows the results from the same model fit to the Mrk 231 image. The third panel shows the radial profile for the Mrk 231 image, constant background subtracted and normalized to the peak value of the PSF model for the Mrk 231 image. Overlaid is the PSF model for the PSF observation. The radial profile of the PSF is narrower than the Mrk 231 PSF, indicating extended emission. The bottom panel shows the radial profile of Mrk 231 and model for a fit using a single Gaussian model convolved with the background-subtracted observed PSF image, and the model PSF image. The FWHM of the Gaussian model is $\sim 3.4$ pixels, corresponding to $\sim 40$ pc. Top panel: 
is intrinsically weak and minimally absorbed, as proposed by Yan et al. (2015), or dramatically absorbed, as proposed by Leighly et al. (2015).

The Yan et al. (2015) model assumed that the broad line region emission in Mrk 231 is powered by the photoionizing continuum produced by thindisk accretion onto the smaller of the two black holes. Thus, in order to produce emission lines in the near infrared that are seen to have typical equivalent widths with respect to the observed continuum the equivalent widths with respect small-black-hole-mass continuum would have to be huge, approximately 100 times larger than normal, since the photoionizing continuum extrapolated into the infrared is $\sim 100$ times weaker than the observed continuum (3.2). This seems quite implausible, even without quantitative analysis, and thus provides the first piece of evidence that the Yan et al. (2015) model is untenable.

Using the photonionization code Cloudy, we first established that we were able to produce the observed $\mathrm{He} \mathrm{I}^{*}$ equivalent widths, and $\mathrm{He} \mathrm{I}^{*} / \mathrm{P} \beta$, $\mathrm{P} \alpha / \mathrm{P} \beta$, and $\mathrm{He} \mathrm{I}^{*} / \mathrm{C}$ IV ratios of a comparison sample of objects using two spectral energy distributions that roughly bracket the properties of observed AGN and quasar continua. We next investigated spectral energy distributions similar to those proposed by Yan et al. (2015), i.e., sum-of-blackbodies accretion disks with small inner radii $\left(r_{i n}=3.5 r_{g}\right.$ and Schwartzschild) that were normalized to the observed far-UV continuum. We required the X-ray portion of the spectrum to go through the observed spectrum presented by Teng et al. (2014), a departure from Yan et al. (2015), who neglected the X-ray emission. Mrk 231 has a rather typical He I* $/ \mathrm{P} \beta$ ratio, so we sought simulations that produced both a typical range of $\mathrm{He} \mathrm{I}^{*} / \mathrm{P} \beta$ ratios and the observed intensity of the He $\mathrm{I}^{*}$ emission line. There were none, even if the broad line region is assumed (unrealistically) to fully cover the continuum emitting source. This provided the second piece of evidence that Yan et al. (2015) model is untenable.

Our Cloudy models showed that the He I*/C IV ratio is sensitive to the SED shape, being lower for harder (X-ray bright) SEDs and higher for softer (X-ray weak) SEDs, with the maximum values produced by the SED from the intrinsically Xray weak quasar PHL 1811. However, Mrk 231's He I*/C IV ratio was $\sim 100$ times higher than the one from PHL 1811. We conclude that we do not see the direct C IV emission in Mrk 231. The origin of the emission that we do observe is not known; we speculate it may be due to scattering (7.6), or it may be produced in the BAL outflow (Veilleux et al. 2013, 2016).

Analysis of the 2-D image from archival HST STIS observation shows that the spatial profile of the far-UV continuum is asymmetrically extended. Moreover, the spatial profile in the Mg II and lowexcitation Fe II troughs is similarly extended, suggesting that these absorption lines are saturated and the troughs are filled in by the far-UV continuum. Evidence for extended emission in the far-UV was also found in an archival HST FOC image. The scale of the offset emission in both cases was $\sim 40$ pc.

\subsection{Suggestive Results Regarding the Near Infrared Emission Lines}

The focus of this paper is a critique of the Yan et al. (2015) model, and we do not purport to provide a full model of the broad line region. Nevertheless, we have learned a few interesting things about the infrared lines and Cloudy models for them. The observed $\mathrm{P} \alpha / \mathrm{P} \beta$ line ratios in the comparison sample are rather low, with a range between 0.94 and 1.74 and a mean of 1.2. They are much lower than the Case A (Case B) values of $2.33(2.28)$ in the low density limit (Osterbrock \& Ferland 2006). This suggests that the hydrogen-line emission regions are very optically thick and thermalization is important. This is not unexpected, as it has been known for many years that the $\mathrm{Ly} \alpha / \mathrm{H} \beta$ is observed to be typically around 10 , rather than 23 or 34 (the low and high density Case B limits; Osterbrock \& Ferland 2006). It was surprising, however, to see how thermalization influences line ratios at different densities to produce a strongly localized density range for the simple one-zone model. While arguably not directly applicable to AGN spectra, it may be useful to consider this behavior for complete models of the broad line region.

Also intriguing is the sensitivity of the $\mathrm{He} \mathrm{I}^{*} / \mathrm{P} \beta$ ratio to the SED. On the face of it, this result is not surprising, given that the $\mathrm{He} \mathrm{I}^{*}$ line responds to the strength of the helium continuum, while the $\mathrm{P} \beta$ depends the ionizing flux and column density of the gas. This suggests that the near-infrared 
broad line ratios could be used to infer the intrinsic spectral energy distribution of obscured quasars. But this result must be viewed with extreme caution. In this paper, we used a one-zone model for simplicity, and it is not clear that the same effect would be present in a extended-BLR model. Indeed, it is not clear that these lines can be trivially modeled using an extended-BLR model. Ruff et al. (2012) investigated an LOC model for the hydrogen lines, and found themselves forced into a small region of photoionization parameter space. This could be because the standard LOC, which was historically proposed to explain the high-ionization UV lines (Baldwin et al. 1995), is simply more appropriate for that regime, and less appropriate for the low-ionization lines considered here. In fact, the LOC doesn't always work well for high-ionization lines (Dhanda et al. 2007). In addition, the standard LOC model has a substantial fraction of optically thin clouds, while we have shown that high optical depths are needed to produce sufficient line emission and correct line ratios. Moreover, it is not clear that the SED that illuminates low-ionization-line emitting gas is the same as the continuum we see; it may have been "filtered" by gas producing the high-ionization lines (Leighly 2004). Alternative models using radiation pressure confinement may work better for these lines (Baskin et al. 2014). While this is an interesting and potentially important problem given the dearth of SED diagnostics the infrared, it is beyond the scope of the present paper.

\subsection{Powering the Mid-Infrared Contin- uum}

While we have demonstrated that the strength of the near-IR broad-line emission cannot be accounted for by the UV-to-optical SED as observed, implying the presence of an intrinsically much more luminous ionizing continuum, there is an additional argument to be made based on considerations of the mid-infrared power of Mrk 231 that is independent of the physics of the broadline region. The thermal near- and mid-infrared $(2-20 \mu \mathrm{m})$ emission of quasars is attributed to dust heated by absorption of optical-through$\mathrm{X}$-ray emission from the central engine. The tight, linear correlation between unobscured optical $(0.1-1 \mu \mathrm{m})$ and infrared $(1-100 \mu \mathrm{m})$ quasar luminosities seen in radio-quiet quasars supports this interpretation (e.g., Gallagher et al. 2007). The infrared luminosity is therefore arguably a more robust indicator of bolometric luminosity than the optical-UV as observed because the infrared is much less susceptible to dust extinction. Gallagher et al. (2007) recommended the $3 \mu \mathrm{m}$ luminosity in particular as a good single value for estimating quasar bolometric luminosities because the hottest dust is certainly powered by the AGN with no starburst contamination. The weak PAH emission seen in the $L$-band and midinfrared spectra of Mrk 231 supports the claim that this region of the spectrum is dominated by the quasar Imanishi et al. 2007; Weedman et al. 2005). From the $L$-band spectrum of Mrk 231 presented in Imanishi et al. (2007), the $L_{3 \mu \mathrm{m}}$ is $7.5 \times 10^{44} \mathrm{erg} \mathrm{s}^{-1}$. Using the $3 \mu \mathrm{m}$-to-IR bolometric correction of $3.44 \pm 1.68$ (Gallagher et al. 2007) gives $L_{\mathrm{IR}}=(3.86 \pm 1.26) \times 10^{45} \mathrm{erg} \mathrm{s}^{-1}$. This is more than an order of magnitude greater than the $0.1-1 \mu \mathrm{m}$ luminosity of the observed continuum (gray dashed + green dashed continua) shown in Figure 2: $L_{\mathrm{opt} \text {,obs }}=2.1 \times 10^{44} \mathrm{erg} \mathrm{s}^{-1}$. Correcting for SMC extinction with $E(B-V)=0.1$ following Yan et al. (2015) brings $L_{\text {opt,obs up }}$ to $2.9 \times 10^{44} \mathrm{erg} \mathrm{s}^{-1}$, still well below the $L_{\mathrm{IR}}$ that is supposedly powered by this continuum. However, the extinction-corrected $0.1-1.0 \mu \mathrm{m}$ continuum (orange dashed line in Fig. 2) gives $L_{\text {opt }}=2.7 \times 10^{45} \mathrm{erg} \mathrm{s}^{-1}$, in line with the expectations from the IR power (from Figure 2 of Gallagher et al. 2007).

\subsection{Polarization}

The Yan et al. (2015) model also does not adequately explain the near-UV-to-optical polarization in Mrk 231, which is significant and strongly rising to the blue through $\sim 3000 \AA$ (Smith et al. 1995). This kind of polarization signature is commonly seen in reddened objects. Wills et al. (1992) analyzed the similarly-polarized infraredluminous quasar IRAS $13349+2438$. They showed that electron scattering, which produces a wavelengthindependent polarization, combined with a reddened continuum produces a blue-polarized spectrum. That is, the intrinsic polarization is constant, but appears to increase toward the blue due to dilution by the unpolarized, reddened, direct continuum in the red. Alternatively, scattering by small dust grains pro- 
duces polarization increasing toward the blue (Rayleigh scattering). A similar polarization signature is seen among many Type 1 reddened objects (e.g., Smith et al. 2000; Schmid et al. 2001; Hines et al. 2001; Smith et al. 2002b,a, 2003). Mrk 231 is a low-ionization broad absorption line quasar, and these objects are known to be significantly polarized (e.g., Hines \& Wills 1995; Ogle et al. 1999; Schmidt \& Hines 1999; Brotherton et al. 2001; DiPompeo et al. 2011) and reddened (e.g., Reichard et al. 2003; Dai et al. 2008; Krawczyk et al. 2015). In addition, Mrk 231 shows evidence for X-ray absorption (Teng et al. 2014), and polarized Type 1 objects are more likely to suffer X-ray absorption than unpolarized ones (Leighly et al. 1997).

\subsection{Strategic Absorption}

Yan et al. (2015) noted that Mrk 231 has strong optical Fe II emission, and therefore might be expected to have comparably strong UV Fe II emission, which is not seen. The Leighly et al. (2014) circumstellar absorption model explains this lack of strong UV Fe II naturally via reddening; both the continuum and the line emission are attenuated in the near UV (Fig. 21). Since Yan et al. (2015) inferred that reddening is minimal, they proposed another explanation for the weak UV Fe II emission, that since Mrk 231 is a known Fe II absorption line quasar (FeLoBAL, Smith et al. 1995), the Fe II emission is absorbed exactly by the Fe II absorption in the BAL outflow. This idea is untenable for several reasons. First, the velocity offset of the low-ionization line absorption in Mrk 231 is known to be between about $-5,500$ and $-4,000 \mathrm{~km} \mathrm{~s}^{-1}$ (e.g., Figure 8 in Leighly et al. (2014)). Yan et al. (2015) required a much broader velocity width to produce their exact subtraction, between $-8,000$ and $-1000 \mathrm{~km} \mathrm{~s}^{-1}$.

More importantly, however, the recent HST STIS observation (which appears very similar to the spectrum shown in Fig. 2, albeit with the significant advantage of better signal-to-noise ratio and resolution) shows that Mrk 231's nearUV Fe II absorption is strong, with saturated low-excitation Fe II from levels between 0 and $0.12 \mathrm{eV}$ (near 2600 and $2400 \mathrm{~A}$ ), while absorption from higher excitation levels between 0.98 and $1.1 \mathrm{eV}$ (near $2750 \AA$ ) is present but weaker
(Veilleux et al. 2016). Some of the Leighly et al. (2014) solutions are therefore ruled out based on the presence of the higher excitation Fe II, which requires a higher density (e.g., Lucy et al. 2014, Fig. 13). But some of the optimized models discussed in $\S 8$ of Leighly et al. (2014) produce sufficient higher excitation Fe II, in particular, the density step-function models with an illuminatedface density $\log n=5.5\left[\mathrm{~cm}^{-3}\right]$, constant pressure in the $\mathrm{H}$ II region, and increasing by a factor of 25 in the partially ionized zone. As seen in Fig. 12 in Leighly et al. (2014), the inferred location of these optimized models is 40 parsecs, somewhat interior to the nuclear starburst, but interestingly consistent with the extended emission discussed in $\S 6$. Whether or not these models fit the new STIS data in detail is beyond the scope of the current paper.

\subsection{The Origin of the Resolved FUV Emission}

In 96 we describe analysis of archival STIS and FOC data that indicates the presence of extended far UV continuum emission. The most convincing evidence comes from the discovery that the spatial profile is broader and offset in the broad absorption troughs (e.g., Mg II) compared with the nearUV continuum. The FOC image suffers from poor signal-to-noise ratio and a complicated PSF, but is intriguing as the size scale derived $(\sim 40$ parsecs) is basically the same as that derived from the STIS spatial profile analysis $>36.5$ parsecs, and a revised estimate of the location of the absorber.

One of the mysterious properties of the far UV spectrum is the lack of broad absorption lines, despite the presence of strong optical, near-UV, and infrared absorption lines. This can not be a consequence of a special combination of photoionization gas parameters. The presence of Fe II absorption means that the column density of the absorber extends beyond the hydrogen ionization front (e.g., Lucy et al. 2014). The presence of strong He I* absorption sets a lower limit on the ionization parameter (Leighly et al. 2011). Strong absorption from many species would be expected, including, for example, C IV.

Veilleux et al. (2013, 2016) explain the lack of far UV absorption lines via a special geometry (specifically, Veilleux et al. 2016, Fig. 8). They postulate a dusty outflow that covers the optical 
and near UV emission region, producing absorption lines on that continuum. It is opaque to the far UV, but $10 \%$ reaches the viewer unobscured. The important point, for this discussion, is that the FUV continuum is thought to be very compact, emitted by the funnel of a slim disk, and it would be expected to be unresolved.

The presence of extended emission casts some doubt upon this explanation. Our analysis shows that a significant amount of FUV continuum is not coincident with the central engine emitting the near-UV and optical continuum. It is possible that all of the far-UV continuum emerging from the central engine is attenuated by the significant reddening that is the origin of the rolloff in the optical and UV. Then, the observed far-UV continuum comes from somewhere near the central engine, but not from the central engine, and that is why there are no far-UV broad absorption lines.

UV variability is commonly observed in AGN and quasars, and it can be used to identify them (e.g., Peters et al. 2015; Morganson et al. 2014). An origin of the far UV continuum in extended emission in Mrk 231 predicts that the continuum should not be variable. Veilleux et al. (2016) report no significant FUV variability between two COS observations separated by 3 years. The lack of variability provides further support for dominance of an extended emission component for the far UV continuum.

The offset emission may be somewhat similar to an optical continuum peak or "hot spot" in the inner narrow-line region of the nearby Seyfert 2 galaxy NGC 1068. Long-slit STIS spectroscopy revealed a continuum shape indistinguishable from Seyfert 1 galaxies, as well as broad components of emission lines such as C IV. Crenshaw \& Kraemer (2000) conclude that this component is reflected emission from the central engine. The hot spot lies $30 \mathrm{pc}$ from the estimated position of the central engine in NGC 1068 Kraemer \& Crenshaw 2000), intriguingly close to the estimates of the location of the extended emission in Mrk 231. An important difference, from a data analysis point of view, is that NGC 1068 is much nearer than Mrk 231; e.g., an arcsecond corresponds to 60 parsecs in NGC 1068 versus 867 parsecs in Mrk 231.

We suggest that a scattering/reflection scenario may have been too hastily dismissed by
Veilleux et al. (2013). The continuum flux near $2000 \AA$ is found to be about 165 times weaker than the intrinsic continuum inferred by Leighly et al. (2014), i.e., $0.6 \%$ of the intrinsic flux. Such a level of scattering is not implausible; e.g., a median scattering efficiency of $2.3 \%$ is found in a sample of obscured quasars (e.g., Obied et al. 2015, although the scattering regions in those galaxies are very large). Veilleux et al. (2013) dismiss scattering due to the low level of polarization in the UV, as observed by Smith et al. (1995), but we note that while the polarization in the UV is lower than it is in the near-UV, it is not zero. Moreover, it shows a position angle rotation compared with the strong near-UV polarization, which suggests a different origin. Also, we note that the degree of polarization that is observed depends on the asymmetry of the scatterer. For example, the hot spot in NGC 1068 shows a high degree of polarization, but the geometry in that case (a relatively small, single reflector well resolved from the hidden nucleus) is nearly ideal for producing high polarization. If the scatterer in Mrk 231 has a large solid angle to the nucleus, high polarization is not expected. In addition, the optical polarization in Mrk 231 has been observed to be variable (Gallagher et al. 2005), and the HST UV polarization observation was performed more than 20 years ago.

Finally, an intriguing possibility is that the far UV continuum may be nuclear continuum reflected from the wind on the far side of the nucleus. This is suggested by the confluence of distance estimates: 36-40 parsecs for the extended emission (§ 60), and $\sim 40$ parsecs as a revised approximate distance to the broad absorption line gas (§ 7.5).

KML acknowledges useful conversations with Dick Henry, Mike Brotherton, and Jerry Kriss. She also thanks Mike Crenshaw for reviewing the imaging/spatial analysis section, and suggesting the intriguing parallel with NGC 1068, and the anonymous referee for suggestions that improved the content and clarity of the paper. ABL thanks Zoltan Haiman for useful comments. The authors gratefully acknowledge Jules Halpern for the title suggestion. KML thanks Erin Cooper for processing the Mrk 231 spectra. KML acknowledges support through STScI HST-GO-14058.001-A. SCG thanks the Natural Science and Engineering Re- 
search Council of Canada for support.

Facilities: IRTF (SpeX), HST (STIS, FOC)

\section{REFERENCES}

Baldwin, J., Ferland, G., Korista, K., \& Verner, D. 1995, ApJ, 455, L119

Barrett, P. E., \& Dressel, L. L. 2006, in The 2005 HST Calibration Workshop: Hubble After the Transition to Two-Gyro Mode, ed. A. M. Koekemoer, P. Goudfrooij, \& L. L. Dressel, 260

Baskin, A., Laor, A., \& Stern, J. 2014, MNRAS, 438,604

Bentz, M. C., Denney, K. D., Grier, C. J., et al. 2013, ApJ, 767, 149

Bottorff, M., Ferland, G., Baldwin, J., \& Korista, K. 2000, ApJ, 542, 644

Brotherton, M. S., Arav, N., Becker, R. H., et al. 2001, ApJ, 546, 134

Casebeer, D. A., Leighly, K. M., \& Baron, E. 2006, ApJ, 637, 157

Crenshaw, D. M., \& Kraemer, S. B. 2000, ApJ, 532,247

Crenshaw, D. M., Kraemer, S. B., Bruhweiler, F. C., \& Ruiz, J. R. 2001, ApJ, 555, 633

Dai, X., Shankar, F., \& Sivakoff, G. R. 2008, ApJ, 672,108

Davidson, K., \& Netzer, H. 1979, Reviews of Modern Physics, 51, 715

Denney, K. D., De Rosa, G., Croxall, K., et al. 2014, ApJ, 796, 134

Dhanda, N., Baldwin, J. A., Bentz, M. C., \& Osmer, P. S. 2007, ApJ, 658, 804

Dietrich, M., Hamann, F., Shields, J. C., et al. 2002, ApJ, 581, 912

DiPompeo, M. A., Brotherton, M. S., \& De Breuck, C. 2011, ApJS, 193, 9

Farrah, D., Afonso, J., Efstathiou, A., et al. 2003, MNRAS, 343, 585
Ferland, G. J., Porter, R. L., van Hoof, P. A. M., et al. 2013, Rev. Mexicana Astron. Astrofis., 49, 137

Francis, P. J., Hewett, P. C., Foltz, C. B., et al. 1991, ApJ, 373, 465

Frank, J., King, A., \& Raine, D. J. 2002, Accretion Power in Astrophysics: Third Edition

Fynbo, J. P. U., Krühler, T., Leighly, K., et al. 2014, A\&A, 572, A12

Gallagher, S. C., Brandt, W. N., Chartas, G., Garmire, G. P., \& Sambruna, R. M. 2002, ApJ, 569,655

Gallagher, S. C., Richards, G. T., Lacy, M., et al. 2007, ApJ, 661, 30

Gallagher, S. C., Schmidt, G. D., Smith, P. S., et al. 2005, ApJ, 633, 71

Glikman, E., Helfand, D. J., \& White, R. L. 2006, ApJ, 640, 579

Goobar, A. 2008, ApJ, 686, L103

Hamann, F., Kanekar, N., Prochaska, J. X., et al. 2011, MNRAS, 410, 1957

Hines, D. C., Schmidt, G. D., Gordon, K. D., et al. 2001, ApJ, 563, 512

Hines, D. C., \& Wills, B. J. 1995, ApJ, 448, L69

Imanishi, M., Nakanishi, K., Tamura, Y., Oi, N., \& Kohno, K. 2007, AJ, 134, 2366

Koratkar, A., \& Blaes, O. 1999, PASP, 111, 1

Korista, K., Baldwin, J., Ferland, G., \& Verner, D. 1997, ApJS, 108, 401

Kraemer, S. B., \& Crenshaw, D. M. 2000, ApJ, 532,256

Krawczyk, C. M., Richards, G. T., Gallagher, S. C., et al. 2015, AJ, 149, 203

Kriss, G. A., Peterson, B. M., Crenshaw, D. M., \& Zheng, W. 2000, ApJ, 535, 58

Kuraszkiewicz, J. K., Green, P. J., Crenshaw, D. M., et al. 2004, ApJS, 150, 165

Kuraszkiewicz, J. K., Green, P. J., Forster, K., et al. 2002, ApJS, 143, 257 
Kwan, J., \& Krolik, J. H. 1981, ApJ, 250, 478

Landt, H., Bentz, M. C., Ward, M. J., et al. 2008, ApJS, 174, 282

Laor, A., Bahcall, J. N., Jannuzi, B. T., et al. 1994, ApJ, 420, 110

Leighly, K. M. 2004, ApJ, 611, 125

Leighly, K. M., Cooper, E., Grupe, D., Terndrup, D. M., \& Komossa, S. 2015, ApJ, 809, L13

Leighly, K. M., Dietrich, M., \& Barber, S. 2011, ApJ, 728, 94

Leighly, K. M., Halpern, J. P., Helfand, D. J., Becker, R. H., \& Impey, C. D. 2001, AJ, 121, 2889

Leighly, K. M., Halpern, J. P., Jenkins, E. B., \& Casebeer, D. 2007a, ApJS, 173, 1

Leighly, K. M., Halpern, J. P., Jenkins, E. B., et al. 2007b, ApJ, 663, 103

Leighly, K. M., Hamann, F., Casebeer, D. A., \& Grupe, D. 2009, ApJ, 701, 176

Leighly, K. M., Kay, L. E., Wills, B. J., Wills, D., \& Grupe, D. 1997, ApJ, 489, L137

Leighly, K. M., Terndrup, D. M., Baron, E., et al. 2014, ApJ, 788, 123

Lucy, A. B., Leighly, K. M., Terndrup, D. M., Dietrich, M., \& Gallagher, S. C. 2014, ApJ, 783, 58

Morganson, E., Burgett, W. S., Chambers, K. C., et al. 2014, ApJ, 784, 92

Natali, F., Giallongo, E., Cristiani, S., \& La Franca, F. 1998, AJ, 115, 397

Obied, G., Zakamska, N. L., Wylezalek, D., \& Liu, G. 2015, ArXiv e-prints

O'Brien, P. T., Reeves, J. N., Simpson, C., \& Ward, M. J. 2005, MNRAS, 360, L25

Ogle, P. M., Cohen, M. H., Miller, J. S., et al. 1999, ApJS, 125, 1

Osterbrock, D. E., \& Ferland, G. J. 2006, Astrophysics of gaseous nebulae and active galactic nuclei
Pei, Y. C. 1992, ApJ, 395, 130

Peters, C. M., Richards, G. T., Myers, A. D., et al. 2015, ApJ, 811, 95

Reichard, T. A., Richards, G. T., Hall, P. B., et al. 2003, AJ, 126, 2594

Richards, G. T., Lacy, M., Storrie-Lombardi, L. J., et al. 2006, ApJS, 166, 470

Riffel, R., Rodríguez-Ardila, A., \& Pastoriza, M. G. 2006, A\&A, 457, 61

Ruff, A. J., Floyd, D. J. E., Webster, R. L., Korista, K. T., \& Landt, H. 2012, ApJ, 754, 18

Rupke, D. S. N., \& Veilleux, S. 2011, ApJ, 729, L27

Sanders, D. B., Soifer, B. T., Elias, J. H., Neugebauer, G., \& Matthews, K. 1988, ApJ, 328, L35

Schmid, H. M., Appenzeller, I., Camenzind, M., et al. 2001, A\&A, 372, 59

Schmidt, G. D., \& Hines, D. C. 1999, ApJ, 512, 125

Selsing, J., Fynbo, J. P. U., Christensen, L., \& Krogager, J.-K. 2015, ArXiv e-prints

Smith, J. E., Young, S., Robinson, A., et al. 2002a, MNRAS, 335, 773

Smith, P. S., Schmidt, G. D., Allen, R. G., \& Angel, J. R. P. 1995, ApJ, 444, 146

Smith, P. S., Schmidt, G. D., Hines, D. C., Cutri, R. M., \& Nelson, B. O. 2000, ApJ, 545, L19

—. 2002b, ApJ, 569, 23

Smith, P. S., Schmidt, G. D., Hines, D. C., \& Foltz, C. B. 2003, ApJ, 593, 676

Steffen, A. T., Strateva, I., Brandt, W. N., et al. 2006, AJ, 131, 2826

Teng, S. H., Brandt, W. N., Harrison, F. A., et al. 2014, ApJ, 785, 19

Tilton, E. M., \& Shull, J. M. 2013, ApJ, 774, 67

Vanden Berk, D. E., Richards, G. T., Bauer, A., et al. 2001, AJ, 122, 549 
Veilleux, S., Melendez, M., Tripp, T. M., Hamann, F., \& Rupke, D. S. N. 2016, ArXiv e-prints

Veilleux, S., Trippe, M., Hamann, F., et al. 2013, ApJ, 764, 15

Wang, L. 2005, ApJ, 635, L33

Wang, T.-G., Lu, Y.-J., \& Zhou, Y.-Y. 1998, ApJ, 493,1

Weedman, D. W., Hao, L., Higdon, S. J. U., et al. 2005, ApJ, 633, 706

Wills, B. J., Wills, D., Evans, II, N. J., et al. 1992, ApJ, 400, 96

Witt, A. N., Thronson, Jr., H. A., \& Capuano, Jr., J. M. 1992, ApJ, 393, 611

Yan, C.-S., Lu, Y., Dai, X., \& Yu, Q. 2015, ApJ, 809, 117

This 2-column preprint was prepared with the AAS IATEX

macros v5.2. 